\title{
LA CORTE VICEREALE DI SICILIA TRA PUBBLICO E PRIVATO: DINAMICHE CORTIGIANE, RUOLI, POTERI
}

\author{
Rossella Cancila \\ (Università di Palermo) \\ rossella.cancila@unipa.it
}

\section{RIASSUNTO}

Il saggio delinea alcuni caratteri della corte del viceré in Sicilia in età asburgica: la sua famiglia, la cerchia dei confidenti, composizione e articolazione dei ruoli istituzionali, costi, forme del coinvolgimento. Ne emerge un contesto articolato, uno scenario in cui si confrontavano poteri di diversa intensità e in competizione sul territorio, si determinavano scontri giurisdizionali, dispute patrimoniali e accordi matrimoniali, liti e vendette private: elementi che evidenziano la complessità del rapporto fra nobiltà periferica e potere centrale, relazioni interpersonali e reti di livello internazionale, pratiche negoziali diffuse, che confermano la rappresentazione di un regno niente affatto passivo alla volontà di Madrid.

PAROLE CHIAVE: corte; viceré; Sicilia; Monarchia spagnola; ruoli; poteri.

\section{THE VICEROY'S COURT IN SICILY BETWEEN PUBLIC AND PRIVATE: COURTESAN DYNAMICS, ROLES, POWERS}

\begin{abstract}
The essay outlines some characteristics of the viceroy's court in Sicily during the Habsburg Age: his family, the circle of confidants, composition and articulation of institutional roles, costs, forms of participation. What emerges is a articulated context - powers of different intensity and in competing on the territory, jurisdictional conflicts, property disputes and matrimonial agreements, private vendettas - elements that highlight the complexity of the relationship between peripheral nobility and central power, interpersonal relationships and international networks, pervasive negotiation process, which confirm the representation of a kingdom not entirely subject to the will of Madrid.
\end{abstract}

KEY WORDS: court; viceroy; Sicily; Spanish Monarchy; roles; powers. 
Il tema della corte vicereale di Sicilia non è mai stato organicamente affrontato, sebbene non manchino pregevoli studi sui poteri del viceré, alter ego del sovrano, primo testimone sul territorio della dignità reale ${ }^{1}$, così come su singole personalità ${ }^{2}$, di cui di volta in volta si è analizzata la capacità di governo, il rapporto con le élites siciliane e le istituzioni locali, le strategie di ricerca del consenso e i conflitti, le importanti relazioni internazionali. Si è scritto sui cortei che accompagnavano il suo ingresso a Palermo, e dunque sulle cerimonie e sulle feste religiose e civili che scandivano la vita pubblica negli anni della sua permanenza nel regno. Sappiamo come si disponeva la cerimonialità siciliana attorno a un viceré in relazione alle diverse circostanze e alla diversa presenza di soggetti in campo; conosciamo dispute cerimoniali specchio di relazioni politiche e istituzionali spesso controverse $e^{3}$.

La più recente storiografia ha inoltre evidenziato l'importanza di collegare le dinamiche locali con forze e interessi che si muovevano sul piano sovralocale, individuando reti gerarchiche di potere all'interno della compagine spagnola, ma anche

$*$ Abbreviazioni: ASPa $=$ Archivio di Stato di Palermo; ASCPa $=$ Archivio storico del Comune di Palermo; ASTO $=$ Archivio di Stato di Torino $; \mathrm{BCPa}=$ Biblioteca Comunale di Palermo (Casa Professa).

${ }^{1}$ Per la Sicilia vanno segnalati i lavori di Camillo Giardina, L'istituto del Viceré di Sicilia (1415-1798) (Palermo: Boccone Del Povero, 1930); Helmut G. Koenigsberger, L'esercizio dell'impero (Palermo: Sellerio, 1997); Domenico Ligresti, Sicilia aperta. Mobilità di uomini e idee nella Sicilia spagnola (secoli XV-XVII) (Palermo: Quaderni di Mediterranea-ricerche storiche, 2006). Riferimento imprescindibile rimane Giovanni Evangelista Di Blasi, Storia cronologica de' Viceré, edizione a cura di Illuminato Peri (Palermo: Edizioni della Regione Siciliana, 1974).

${ }^{2}$ Segnalo in particolare per la ricchezza documentaria il volume di Nicoletta Bazzano, Marco Antonio Colonna (Roma: Salerno, 2003); e il pionieristico Gaetano Capasso, Il governo di don Ferrante Gonzaga in Sicilia dal 1535 al 1543 (Palermo: Scuola Tip. «Boccone del povero», 1905). Ben condotta anche l'analisi di Valentina Favarò, Carriere in movimento. Francisco Ruiz de Castro e la monarchia di Filippo III (Palermo: Studi e ricerche - Mediterranea-ricerche storiche, 2013). Come Presidenti del regno, cfr. Lina Scalisi, "Magnus Siculus". La Sicilia tra impero e monarchia (1513-1578) (Roma-Bari: Laterza, 2012); Eadem, Da Palermo a Colonia. Carlo Aragona Tagliavia e la questione delle Fiandre (1577-1580) (Roma: Viella, 2019); Rafaella Pilo, Luigi Guglielmo Moncada e il governo della Sicilia (1635-1639) (Caltanissetta-Roma: Sciascia editore, 2008). Si tratta di studi molto attenti a dinamiche e contesti più ampi, che includono le forti proiezioni internazionali di queste personalità. Diversi profili sono inoltre delineati nel volume Stefano Piazza, a cura di, La Sicilia dei viceré nell'età degli Asburgo (1516-1700) (Palermo: Edizioni Caracol, 2016) con un ampio intervallo cronologico e una particolare attenzione agli aspetti urbanistici e architettonici.

${ }^{3}$ In particolare, cfr. Francesco Benigno, La Sicilia dei viceré. Potere e conflitto nella Sicilia spagnola (sec. XVI-XVIII) (Palermo: Palermo University Press, 2017); Nicoletta Bazzano, Palermo fastosissima. Cerimonie cittadine in età spagnola (Palermo: Palermo University Press, 2016). Più recentemente il lavoro di Loris De Nardi, Oltre il cerimoniale dei viceré. Le dinamiche istituzionali nella Sicilia barocca (Padova: libreriauniversitaria.it edizioni, 2014), che però presenta qualche citazione non sempre verificabile. I cerimoniali del Regno di Sicilia si trovano in ASPa, Protonotaro del Regno, Cerimoniale dei viceré, regg. 1060-1067. In particolare, cfr. Cerimoniale de' Signori Vicerè (1584-1688), a cura di Enrico Mazzarese Fardella (Palermo: Società Siciliana per la Storia Patria 1976), contenente l'edizione del registro n. 1060. Per Napoli, rinvio alle edizioni curate da Attilio Antonelli nella serie Cerimoniali della corte di Napoli, 5 Voll. (Napoli: Arte'm, 2012-2019); e anche il più recente Ida Mauro, Spazio urbano e rappresentazione del potere: le cerimonie della città di Napoli dopo la rivolta di Masaniello (1648-1672) (Napoli: FedOAPress, 2020). Per Milano, cfr. Cinzia Cremonini, Alla corte del governatore. Feste, riti e cerimonie a Milano tra XVI e XVIII secolo (Roma: Bulzoni, 2012). 
strategie familiari e fazionali, contesti ampi o più ristretti, in cui le posizioni si mescolavano e si definivano sulla base di fronti, relazioni, schemi che si disponevano in modo fluido e variabile ${ }^{4}$. Palermo era sede di una corte periferica, caratterizzata dall'assenza del re, e come altrove il viceré tendeva a riprodurvi gli equilibri e le dinamiche madrilene; ma è ormai abbastanza chiaro come le periferie fossero poli attivi, capaci di condizionare le decisioni del centro, dove istanze e contrasti prodotti a livello locale inevitabilmente rimbalzavano, producendo effetti conseguenti ${ }^{5}$.

I viceré senza dubbio erano protagonisti di primo piano, grandi personaggi, componenti di prestigiosi casati, esponenti di schieramenti politici, portatori di orientamenti e interessi particolari, da inquadrare all'interno di precisi legami non solo col sovrano, ma anche con altri esponenti della sua corte con cui intessevano relazioni formali e informali e stabilivano alleanze orizzontali e verticali, allo scopo di ottenerne vantaggi per sé, per la propria famiglia, per i propri amici. Ma non erano gli unici attori in campo nei territori, né possono considerarsi dei semplici esecutori di ordini che provenivano da Madrid. La gamma dei poteri formali e informali e delle giurisdizioni territoriali con cui entravano in relazione era vasta; la loro vita pubblica era scandita da incontri, adunate, sedute, riti e cerimonie civili, militari, religiose, occasioni diverse attraverso cui costruivano - pur spesso nella breve durata del loro mandato - alleanze, reciprocità, si procuravano rivalità e inimicizie. Certamente c'erano anche spazi privilegiati di corrispondenza e di contatto informali, in cui confidenti e consiglieri avevano accesso diretto a diverso titolo alla loro persona; o più in generale erano parte di un entourage più ristretto, quasi domestico: ufficiali, militari, segretari, religiosi, artisti, percettori, amici, clienti, servitori, cortigiani in generale. A ogni cambio del vertice, cambiava inevitabilmente la squadra e i posti spendibili venivano spartiti tra i membri del gruppo favorito. Ma questo non è di per sé indice di omogeneità.

La corte era per eccellenza lo spazio delle relazioni, in cui canalizzare le contrapposizioni e consolidare l'integrazione. Certo la corte siciliana non può essere paragonata alla conformazione delle corti europee, né a quelle principesche italiane, che hanno una caratterizzazione piena in termini di sovranità, nella misura in cui si riferiscono a Stati comunque indipendenti. Il sovrano del regno di Sicilia risiedeva a Madrid, era fisicamente "assente", condizione questa comune ad altre aree della Monarchia spagnola, ma era tuttavia presente in ciascuno dei suoi domini sotto altre forme. Regni, stati e capitali rivendicavano autonomia, sovranità e ruoli, ma l'essere sedi di corti provinciali rispetto alla corte centrale di Madrid ne fa un terreno

\footnotetext{
${ }^{4}$ Per una visione complessiva e comparativa dell'istituto vicereale nella Monarchia spagnola, rinvio al volume di Manuel Rivero Rodríguez, La edad de oro de los virreyes: el virreinato en la monarquía hispánica durante los siglos XVI y XVII (Madrid: Ediciones Akal, 2011); Aurelio Musi, L'impero dei viceré (Bologna: il Mulino, 2013); Carlos José Hernando Sánchez, "Los virreyes de la Monarquía española en Italia. Evolución y práctica de un oficio de gobierno", Studia historica. Historia moderna 26 (2004): 43-73. Si veda anche Francesca Cantù, a cura di, Las Cortes Virreinales de la Monarquía Española, (Roma: Viella, 2008).

${ }^{5}$ Francesco Benigno, L'ombra del re. Ministri e lotta politica nella Spagna del Seicento (Venezia: Marsilio, 1992) con particolare riferimento al caso del viceré Osuna.
} 
particolarmente interessante di studio ${ }^{6}$. Nelle pagine che seguono si cercherà di delineare alcuni caratteri della corte del viceré in Sicilia, la sua famiglia, la cerchia dei confidenti, composizione e articolazione dei ruoli istituzionali, costi, forme del coinvolgimento. Seppure nell'estrema sintesi che questa collocazione richiede, si può tentare di offrire un quadro d'insieme, valutando le diverse relazioni che si instauravano attorno al viceré e nella sua corte, assumendo come criterio di analisi la prossimità $\mathrm{o}$, se si preferisce, quello della gerarchia e della distinzione ${ }^{7}$. Ne emerge comunque un contesto articolato, uno scenario in cui si confrontavano poteri di diversa intensità e in competizione sul territorio, scontri giurisdizionali, dispute patrimoniali e accordi matrimoniali, liti e vendette private: elementi che evidenziano il rapporto anche controverso fra nobiltà periferica e potere centrale, relazioni interpersonali e reti di livello internazionale, che confermano la rappresentazione di un regno niente affatto passivo alla volontà di Madrid.

\footnotetext{
${ }^{6}$ Una buona sintesi delle questioni storiografiche e dei nodi problematici è offerta da Antonio Alvarez-Ossorio Alvariño, "Corte y provincia en la Monarquía Católica: la corte de Madrid y el Estado de Milán, 1660-1700", in La Lombardia spagnola: nuovi indirizæi di ricerca, a cura di Elena Brambilla e Giovanni Muto (Milano: Unicopli, 1997), 283-341, che si occupa poi nello specifico del caso milanese. Su Milano vanno segnalati gli studi di Cesare Mozzarelli su Ferrante Gonzaga e di Gianvittorio Signorotto sul marchese di Caracena inseriti nel volume Gianvittorio Signorotto, a cura di, "L'Italia degli Austrias._Monarchia cattolica e domini italiani nei secoli XVI e XVII", numero monografico della rivista Cheiron 17-18 (1992). Cfr. anche Gianvittorio Signorotto, a cura di, Ferrante Gonzaga. Il Mediterraneo, l'Impero (1507-1557) (Roma: Bulzoni, 2009). Per Napoli, cfr. in particolare l'ormai pioneristico Carlos José Hernando Sánchez, Castilla y Nápoles en el siglo XVI. El virrey Pedro de Toledo (Valladolid: Junta de Castilla y León, 1994); ma anche Giovanni Muto, "Capital y Corte en la Nápoles española", Reales Sitios 40 (2003): 3-15; Elisa Novi Chavarria, "Corte e viceré di Napoli nell'età di Filippo IV”, in La Corte de Felipe IV (1621-1665). Reconfiguración de la Monarquía Católica, a cura di José Martínez Millán, Rubén González Cuerva e Manuel Rivero Rodríguez (Madrid: Polifemo, 2018), tomo IV, vol. III, 1307-1333.

${ }^{7}$ La produzione sul tema della corte è rilevante tanto da rendere impossibili citazioni esaurienti anche per le molteplici prospettive in cui è stato declinato. Numerosissimi gli studi che si riferiscono ad aspetti particolari della Corte di Madrid prodotti a cura dello IULCE. Segnalo per una sintesi rapida ed efficace delle questioni e delle definizioni il saggio di José Martínez Millán, "La corte de la Monarquía Hispánica", Studia historica. Historia moderna 28 (2006): 17-61. Assai utile per una definizione del termine "corte" nelle sue diverse connotazioni, ma anche per l'impianto storiografico, è Marcello Fantoni, "La corte", in Le parole che noi usiamo: categorie storiografiche e interpretative dell'Europa moderna, a cura di Amedeo Quondam e Marcello Fantoni (Roma: Bulzoni, 2008), 109-141. Sul piano della loro importanza nel quadro del rinnovamento storiografico vanno segnalati gli studi di Cesare Mozzarelli e del gruppo di studiosi raccolti intorno al Centro Studi Europa delle Corti. Si veda in particolare Cesare Mozzarelli e Gianni Venturi, a cura di, L'Europa delle corti alla fine dell'antico regime (Roma: Bulzoni, 1991); ma anche il più recente Marcello Fantoni, a cura di, The Court in Europe (Roma: Bulzoni, 2012). Per una messa a punto storiografica, cfr. Sergio Bertelli, "La corte come problema storiografico. A proposito di alcuni libri (più o meno) recenti", Archivio Storico Italiano 164/1 (2006): 129-163; ma anche Maria Antonietta Visceglia, "Corti italiane e storiografia europea. Linee di lettura", in L'Italia alla fine del Medioevo: $i$ caratteri originali nel quadro europeo, a cura di Federica Cengarle (Firenze: Firenze University Press, 2006), II, 37-85.
} 


\section{LA FAMIGLIA DEL VICERÉ TRA PUBBLICO E PRIVATO}

Non è sempre facile allo stato attuale delle ricerche poter inquadrare solidamente i diversi livelli su cui si reggeva l'impianto cortigiano in Sicilia. Mancano ad esempio studi organici - anche per la difficoltà nel reperimento delle fonti - sulle viceregine come pure sulle donne presenti a corte, che ne costituivano il seguito, che possano in qualche modo far luce sulla loro personalità, sul loro ruolo e sui margini di intervento che avevano a disposizione ${ }^{8}$.

La presenza della viceregina aveva evidenti ricadute non solo in relazione all'organizzazione domestica e alla sistemazione degli alloggi, ma anche rispetto alla socialità: dame di compagnia, organizzazione di feste, partecipazione a cerimonie pubbliche e private, culto dei santi e riti sacri, sono tutti aspetti che la ponevano in un ruolo di grande evidenza. E la sua partecipazione a un evento veniva interpretata come un segnale di interesse e di cortesia nei confronti delle autorità che l'avevano promosso. Il suo arrivo, festeggiato con tutti gli onori, mobilitava nobildonne titolate pronte ad accoglierla e in gara per assicurarsi un posto al suo fianco nelle cerimonie pubbliche. Di norma il cerimoniale prevedeva che desse la spalla destra alla moglie del titolo più antico e la sinistra alla moglie del pretore (la «pretoressa») ${ }^{9}$. Le stesse istituzioni le rendevano omaggio, come appare in diverse occasioni, ad esempio quelle previste dal cerimoniale del Senato di Palermo: il giorno di Natale il Senato si recava al Palazzo reale per accompagnare il viceré alla sacra funzione, e al ritorno prima di licenziarsi saliva «ad alto per fare riverenza, per dare buone feste alla signora viceregina $[\ldots]$ facendoli nel medesimo modo riverenza che a Sua Eccellenza averan fatto» ${ }^{10}$. Così anche la mattina della Pasqua, ritornando dopo pranzo qualora non fosse «comoda in quest'ore farsi tal ufficio» ${ }^{11}$. Non era previsto dunque che accompagnasse il marito in queste come in altre funzioni, se non lo desiderava. Il suo ruolo aveva evidentemente una valenza prettamente privata, ma la sua presenza nella vita pubblica della città la rendeva comunque partecipe del complesso gioco di rappresentazioni, che coinvolgeva i diversi attori sociali, ponendola al centro di numerose attenzioni.

Non solo mogli e madri, ne va comunque considerata l'influenza sul proprio consorte. Il viceré Guzmán prima di morire nel 1677 nominò a succedergli la moglie, Eleonora de Moura, marchesa di Castel Rodrigo, affidandole il governo del Regno ${ }^{12}$.

\footnotetext{
${ }^{8}$ Bazzano, Palermo fastosissima, 22-25. Più avanzati sono gli studi per l'area napoletana, per la quale segnalo Mirella Mafrici, a cura di, Alla corte napoletana. Donne e potere dall'età aragonese al viceregno austriaco 1442-1734 (Napoli: Fridericiana editrice universitaria, 2012).

${ }^{9}$ Nardi, Oltre il cerimoniale, 164.

${ }^{10} \mathrm{BCPa}$, ms. Qq D 45: Baldassare Bologna, Cerimoniale della felice città di Palermo, nel quale brevemente si contengono tutti quei buoni officii di complimenti e ceremonie, che per tutto l'anno ed in varie occorrenze il Senato di Palermo in essa città è costumato di fare, Palermo, 1610-1611, c. 55r. Cerimoniali della città si trovano anche in ASCPa, Cerimoniali del Senato.

${ }^{11}$ Ibidem, c. 82r.

12 Adelaide Baviera Albanese, "I ventisette giorni di 'governo' nel Regno di Sicilia di Eleonora De Moura y Moncada marchesa di Castel Rodrigo (16 aprile-13 maggio 1677)", Archivio Storico Siciliano Serie IV 24 (1998): 267-301. Eleonora era figlia di Francesco de Moura y Mello, di origine portoghese,
} 
Avversata da molti, rimase in carica come gubernatrix (questo il titolo che si ritrova nell'intestazione dei suoi atti) solamente ventisette giorni, ma rappresenta - benché si tratti di un caso isolato - il ruolo di primo piano, seppure discreto, che queste donne, spesso appartenenti a casati di rango, svolgevano a sostegno dei propri mariti. È noto l'impegno della moglie del viceré Juan de Vega, Eleonora Osorio, figlia del marchese di Astorga, nel perorare l'ingresso dei gesuiti in Sicilia, dove a Messina nel 1548 fu fondato il loro primo collegio e poi l'anno successivo anche a Palermo. Amici personali di Ignazio di Loyola, che avevano conosciuto a Roma, i due coniugi scelsero il gesuita Gerolamo Domenech come istitutore del figlio e come loro confessore, e accolsero a Palermo Diego Laynez, uno dei fondatori della Compagnia, garantendo all'ordine protezione e favori ${ }^{13}$. Molto influente dovette essere anche Luisa de Sandoval Padilla, figlia di Cristóbal Gómez de Sandoval, I duca di Uceda, e nipote del I duca di Lerma, consorte del viceré Juan Alfonso Enríquez (1641), se il giurista Mario Cutelli, considerandolo «incapaz», con l'evidente intento di screditarlo giunse sino a insinuare che avesse abbandonato nelle mani della moglie e dei suoi corrotti segretari l'attività di governo $^{14}$.

D'altra parte, mogli e mariti potevano essere in affari: il viceré de Vega corseggiava a titolo privato a nome della moglie, imitato qualche anno più tardi dal viceré Juan de la Cerda, duca di Medinaceli, che aggiunse le sue due galeotte alla flotta che nel 1558 cercò invano di prendere Tripoli ${ }^{15}$. A sua volta il genero di Medinaceli, Pietro de Luna andava in corso per suo conto e anche a nome della moglie, se la duchessa di Bivona nel 1570 chiedeva e otteneva di poter armare una galeotta per «mandarla in corso», mentre fervevano i preparativi di Lepanto. Anche il viceré Maqueda tra Cinque e Seicento corseggiava per conto della moglie con due vascelli di 200 tonnellate. Secondo le cronache del tempo la corsa gli procurava «cose di gran prezzo», lane, spezie, drappi d'oro e di seta, gioie, perle, persino «persone di ricattito e di servizio» ${ }^{16}$. Un'attività, quella corsara, vietata ai viceré ${ }^{17}$, ma assai lucrosa, e alla quale non disdegnavano di partecipare altri personaggi loro vicini, come attesta il caso del segretario di Moncada, Iohan del Rio, che impegnava una sua bireme in azioni piratesche.

Ma la corsa non era il solo affare praticato più o meno alla luce del sole dai viceré di Sicilia, capitolo che meriterebbe qualche approfondimento: è certo significativo che alcuni di essi abbiano chiesto al Parlamento la concessione della

mentre la madre era Anna Maria Moncada Aragona e la Cerda. Il nonno era dunque Antonio Moncada, duca di Montalto e principe di Paternò.

13 Corrado Dollo, Modelli scientifici e filosofici nella Sicilia spagnola (Napoli: Guida, 1984), 22. Sull'argomento per una sintesi, cfr. anche John W. O'Malley, I primi Gesuiti (Milano: Vita e pensiero, 1999), 199, 225-226.

${ }^{14}$ Vittorio Sciuti Russi, Mario Cutelli. Una utopia di governo (Catania: Bonanno, 1994), 42.

${ }^{15}$ Cfr. Rossella Cancila, "Corsa e pirateria nella Sicilia della prima età moderna", Quaderni storici 2 (2001): 368-369.

16 Vincenzo Di Giovanni, Del Palermo restaurato (Palermo: Sellerio, 1989), 340-341.

17 Nelle istruzioni date ad Albuquerque nel 1627 si fa espressamente riferimento al divieto di praticare la corsa: ASTO, Sicilia, inv. I, cat. I, mazzo 2, fasc. 62, cap. 19, cc. 23r-v: Istruzioni date al viceré duca di Albucherche nel 1627 per il governo del Regno di Sicilia. Madrid, 30 maggio 1627. 
naturaleza siciliana, senza la quale non sarebbe stato possibile accedere agli uffici riservati per privilegio ai regnicoli. Grazie a tale privilegio conferitogli nel 1518, Ettore Pignatelli ottenne nel 1524 l'ufficio di maestro portulano del regno non solo per sé a vita, ma anche per i figli e i nipoti. Per goderne era però necessario che anch'essi fossero regnicoli, privilegio che il Parlamento prontamente concesse nel 1525 al figlio primogenito Camillo e ai nipoti Ettore, Fabrizio e Geronimo ${ }^{18}$, i quali comunque non ne assunsero mai l'incarico. L'ufficio di maestro portulano, dotato di giurisdizione civile e criminale, era di primaria importanza perché controllava tutto il commercio frumentario, e appare significativo che il viceré lo abbia voluto per sé, condizionando pesantemente gli equilibri del mercato del grano.

La commistione tra attività pubblica e privata era un dato di fatto, che non destava particolari preoccupazioni, se non in caso di illeciti di una certa gravità, di cui in verità non mancano le attestazioni documentarie. I viceré di Sicilia incassavano ad esempio ordinariamente alcune rendite, come dazi sull'esportazione di alcuni prodotti, beni vacanti, beni sottratti ai nemici, multe, ecc., che costituivano una cospicua fonte di guadagno integrativa rispetto allo stipendio percepito. Nel 1610 il sovrano le avocò al Fisco, ricompensando i viceré con un notevole aumento di salario, quintuplicato sino a 24.000 ducati castigliani ${ }^{19}$. Su tale somma essi però dal 1631 pagavano il diritto di mezz'annata, come tutti coloro che detenevano uffici o titoli ${ }^{20}$. A integrazione dello stipendio ricevevano comunque delle indennità e altri introiti per spese personali (aiuti di costa) o segrete, e godevano di franchigie, ma non fu più loro consentito di ricevere degli extra al di fuori del donativo ordinario di 2500 scudi, che il Parlamento puntualmente gli offriva ${ }^{21}$.

Per quanto numerosi e cospicui, gli emolumenti goduti non dovevano essere sufficienti a mantenere l'elevato tenore di vita che il rango comportava. Tanto più che gli incarichi conferiti all'entourage ristretto dei viceré erano totalmente a loro carico. Così non di rado entravano in affari con mercanti e banchieri ${ }^{22}$. Noto è il caso di Ferrante Gonzaga (1535-1546), contro il quale «si cominciarono a scoprire molte fastidiose malignità, perché l'imputavano che havesse parte nel banco, che in quel tempo haveva aperto in Palermo Lorenzo Maona» ${ }^{23}$, fratello del suo segretario particolare Giovanni, e di usarlo per frodare i proventi delle tratte del frumento, oltre che per gestire

18 Antonino Mongitore, Parlamenti generali ordinarij et straordinarj celebrati nel Regno di Sicilia dal 1494 fino al 1658 (Palermo: presso Pietro Bentivegna, 1717), vol. I, 154 e 166.

${ }^{19}$ Joseph Cesino Foglietta, Pragmaticae Regni Siciliae (Panormi 1700), tomo III, titolo III, prammatica I (18 settembre 1610); Giardina, L'istituto del Viceré, 226-229.

20 Sulla mezz'annata, cfr. Pietro Burgarella, "Le carte della «Commissaria di mezz'annata» nell'Archivio di Stato di Palermo", Rassegna degli Archivi di Stato 33 (1973): 331-358.

${ }^{21}$ Si vedano i documenti citati in Il Parlamento del 1612. Atti e documenti, a cura di Vittorio Sciuti Russi (Catania: Bonanno, 1984), 120-121, 124. Anche Istruzioni date al viceré duca di Albucherche, cap. 113, c. 38v (Madrid 30 maggio 1627).

22 Pedro de Cisneros, Relación de las cosas del Reyno de Sicilia, edizione a cura di Vittorio Sciuti Russi (Napoli: Jovene, 1990), 26.

${ }^{23}$ Scipio Di Castro, Avvertimenti a Marco Antonio Colonna quando andò viceré di Sicilia, edizione a cura di Armando Saitta (Roma: Edizioni di Storia e Letteratura, 1959), 44; Antonino Giuffrida, La finanza pubblica_nella Sicilia del '500 (Caltanissetta-Roma: Sciascia editore, 1999), 231-232. 
compartecipazioni in prestiti e cambi con la Regia Corte. Si consideri che spettava al viceré il controllo delle procedure di rilascio della licenza per l'apertura di un banco come anche quelle per la sua liquidazione.

Anche i figli dei viceré potevano occupare ruoli significativi a sostegno della politica paterna. Se le femmine potevano essere maritate - come più avanti si vedrà con esponenti di illustri casati siciliani, radicando così la loro presenza sul territorio; per i maschi si profilavano incarichi spesso di natura militare, che concorrevano alla costruzione del loro brillante cursus bonorum. In particolare, può considerarsi emblematico il caso di Hernando (Fernando) de Vega, che il padre nominò capitano d'arme "a guerra" per il Val di Noto, ma anche vicario e poi presidente del regno quando fu costretto ad allontanarsi. Si era già distinto nelle campagne militari in Africa, come nella quotidianità della lotta contro le incursioni piratesche sulla costa siciliana, quanto bastava per preferirlo a esponenti dell'aristocrazia feudale siciliana e affidargli nel 1552 il controllo del sistema difensivo dell'isola, che proprio in quegli anni si definiva con una sempre maggiore consapevolezza. Sempre a diretto contatto epistolare col padre, Hernando risultò un tassello fondamentale del disegno del Vega di creare una struttura amministrativa decentrata, in collaborazione continua con la sua segreteria e gestita da persone di sua totale fiducia, che gli consentisse non solo un capillare controllo del territorio, ma anche di portare a compimento l'ambizioso progetto di fortificazione dell'isola, sul quale suo figlio fu in grado di esercitare un ruolo strategico primario ${ }^{24}$.

Tra pubblico e privato va ricordato che ovviamente la corte era anche il luogo di incontri amorosi clandestini, intrighi e tradimenti, spesso relazioni stabili: bastardi e veleni, promiscuità sessuali, vendette e complotti sono temi abbastanza ricorrenti nella letteratura e sui quali in molti casi si sono costruiti i profili dei principi rinascimentali ${ }^{25}$. Come quella del viceré Francesco Ferdinando d'Avalos marchese di Pescara (15681571), che «s'incapricciò» di una giovinetta «e se la sollazzava allo spesso» sino a morirne. Il figlio nato dalla relazione si distinse nelle guerre di Fiandra, mentre la donna «fu casata ad un dottore per suo rispetto» ${ }^{26}$. Il marchese viene descritto come un uomo bellissimo, addirittura idolatrato dalle dame per la sua avvenenza, e inviso pertanto a tanti cavalieri. Forse anche per questo fu accusato di essersi dedicato più agli amori privati che alle questioni politiche demandate al suo segretario ${ }^{27}$. Giudizio ingeneroso in verità, tanto più che proprio sotto il suo mandato Filippo II portò a compimento l'importante riforma dei Tribunali del regno di Sicilia, che di fatto esautorò il potere della nobiltà, mentre la creazione dei Percettori sottraeva alla Deputazione del regno la competenza nella riscossione e amministrazione dei donativi ${ }^{28}$. Provvedimenti che

\footnotetext{
24 Antonino Giuffrida, "La fortezza indifesa e il progetto del Vega per una ristrutturazione del sistema difensivo siciliano", in Mediterraneo in armi (secc. XV-XVIII), a cura di Rossella Cancila (Palermo: Quaderni di Mediterranea-ricerche storiche, 2007), 239-243.

${ }^{25}$ Fantoni, "La corte", 121.

${ }^{26}$ Di Giovanni, Del Palermo restaurato, 315.

${ }^{27}$ Di Blasi, Storia cronologica de' Vicerè, II, 160-161.

28 Sui Percettori, cfr. Rossella Cancila, Fisco ricchezza comunità nella Sicilia del Cinquecento (Roma: Istituto Storico Italiano, 2001), 318-337.
} 
suscitarono forti resistenze nei ceti privilegiati, appoggiati peraltro dall'inquisitore Giovanni Bezerra. Fu una fase di intenso scontro politico - combattuto su più fronti e in direzioni che coinvolsero i meccanismi istituzionali al centro come nelle periferie -, e che si protrasse anche negli anni successivi dominati dalla presidenza di Carlo d'Aragona. Evidentemente, per gli avversari screditare il viceré d'Avalos sul piano dei comportamenti privati rappresentava un'arma assai efficace anche sul versante dell'azione politica e della propaganda, tanto più che invece egli si guadagnava fama di uomo irreprensibile e rigoroso, seppure su altri versanti ${ }^{29}$.

Di dominio pubblico fu anche la tresca amorosa del viceré Colonna (15751584) con la giovane Eufrosina Siracusa Valdaura, moglie del barone del Miserendino, Galcerano Corbera: la relazione assunse risvolti politici a seguito dell'assassinio del marito in circostanze che alimentarono sospetti e macchiarono la reputazione del viceré $^{30}$. L'attività di governo di Colonna fu oggetto della visitas di Gregorio Bravo de Sotomayor, che sottopose a rigorose indagini i suoi più stretti collaboratori: anche l'omicidio del barone del Miserendino entrò nell'inchiesta, coinvolgendo il cugino del viceré e suo luogotenente Pompeo Colonna, sospettato di avere impedito l'arresto di uno dei sicari. Ma non è solo che un tassello di un più ampio disegno di sgretolamento del ruolo e dell'autorevolezza di Colonna, costretto a misurarsi con il vuoto umano e politico, che gli si stava creando attorno ${ }^{31}$. La vicenda fu anche utilizzata dal suo ormai ex segretario Pedro Cisneros per screditarlo, con l'accusa di una condotta che offendeva «gravedad y decoro». Fatti e comportamenti privati che dunque inevitabilmente finivano con l'avere risvolti pubblici e politici anche di una certa serietà, e si prestavano a rese dei conti su altri fronti e con altri strumenti.

\section{TESSITURE MATRIMONIALI DI RANGO}

Il matrimonio dei figli era per i viceré una buona occasione per stringere legami con esponenti della nobiltà siciliana nel quadro dell'integrazione dinastica delle aristocrazie nelle élites transnazionali di governo nella Monarchia. L'interesse da parte di Madrid a seguire le vicende dei lignaggi siciliani e le loro strategie matrimoniali e successorie costituisce un fattore di un certo rilievo, comune peraltro a tutte le realtà inserite nella compagine della monarchia spagnola ${ }^{32}$. Ne consegue un determinante e non occasionale controllo sulle aristocrazie provinciali nella più ampia strategia di potere non solo della Corona, ma anche dei gruppi preminenti a corte allo scopo di

${ }^{29}$ Si veda Roberto Zapperi, “Avalos, Francesco Ferdinando, marchese di Pescara”, Diæionario Biografico degli Italiani, vol. 4 (1962), on-line: https://www.treccani.it/enciclopedia/avalos-francescoferdinando-marchese-di-pescara (Dizionario-Biografico)/ (ultima consulta: 08/11/2021).

${ }^{30}$ La vicenda è raccontata nei particolari da Koenigsberger, L'esercizio dellimpero, 199-202.

31 Bazzano, Marco Antonio Colonna, 296-297.

${ }^{32} \mathrm{Si}$ tratta di un tema particolarmente frequentato dalla storiografia, con riferimento in particolare al XVII secolo. Cfr. i diversi casi riportati da Alvarez-Ossorio Alvariño, "Corte y provincia", 305-307. Relativamente al caso napoletano, ne mette in luce gli elementi di criticità Giulio Sodano, "Le aristocrazie napoletane ai tempi di Filippo IV", in La Corte de Felipe IV (1621-1665), tomo IV, vol. 3, 1357-1358. 
stringere legami e alleanze sui territori, evitando al tempo stesso pericolosi rafforzamenti di alcuni casati a livello locale. Le tessiture matrimoniali frequentemente coinvolgevano il viceré in disegni complessi dai quali passavano i destini di interi casati, si ridisegnavano gli schieramenti, si formalizzavano nuove relazioni. La costruzione di una unione costituiva un affare diplomatico, una mediazione vera e propria, che impegnava il viceré in un lavoro di intersezione tra interessi generali e vantaggi particolari, ed era seguita con molto interesse a Madrid.

Si veda a titolo di esempio il caso de Vega, viceré assai radicato in Sicilia anche per la lunga permanenza nell'isola, che non solo pianificò il matrimonio della figlia, ma pure quello delle figlie di sua sorella, marchesa de Montemayor, con altri pretendenti siciliani. Isabella fu così destinata in sposa nel 1552 a Pietro de Luna, privo di mezzi finanziari ma di casato prestigioso proveniente dal regno di Léon e con una buona dotazione feudale ${ }^{33}$. Il Luna era stato già al centro di trame matrimoniali, se Giovanni Aragona Tagliavia lo aveva destinato alla figlia Olivia, mentre lui preferiva invece la cugina Diana Cardona, erede del marchesato di Giuliana e della contea di Burgio, figlia della seconda moglie del Terranova (Beatrice Luna), che caldeggiava fortemente l'unione. La vicenda fu seguita dall'allora viceré Ferrante Gonzaga, che ne aveva informato Carlo V, e conclusa nel 1545 con la promessa di matrimonio di suo figlio Cesare di solo nove anni con Diana tra caroselli, giostre e rappresentazioni teatrali ${ }^{34}$.

Il Luna invece, grazie al suo prestigioso matrimonio con Isabella de Vega e anche al sostegno del Granvelle presso il sovrano, acquisì nel 1554 il titolo di duca di Bivona, che ne faceva il primo titolo del regno (a scapito di Simone II Ventimiglia, conte di Geraci, anche lui tra i pretendenti di Isabella), e ottenne dal suocero una serie di favori, che concorsero a metterne in cattiva luce in Sicilia l'integrità ${ }^{35}$. Il matrimonio - celebrato a Messina in gran pompa - ruppe il legame tra il viceré e i Ventimiglia, potentissima famiglia dell'aristocrazia feudale siciliana da tempo in contrasto con i Luna, spingendoli a schierarsi con quella consistente parte della nobiltà che chiedeva la rimozione del viceré per la sua alterigia e la sua asprezza nei confronti del baronaggio, sino a inviare una delegazione a Londra al principe Filippo d'Asburgo (il futuro Filippo II), novello sposo della regina d'Inghilterra ${ }^{36}$.

La coppia Vega-Luna, dopo un periodo di permanenza a Palermo, visse nel palazzo ducale di Bivona, circondata da una piccola corte di cui facevano parte anche alcune dame spagnole, che da Roma (il padre era stato in quella sede ambasciatore di Carlo V) Isabella aveva portato con sé, come Maria de Messa, Maria Usorio e Imperia

33 Scalisi, "Magnus Siculus", 117-118.

34 Ibidem, 44. Diana partì con la famiglia Gonzaga, ma rientrò dalla madre e sposò infine Vespasiano Gonzaga, che sospettandola di tradimento la uccise. Olivia invece si ritirò a Castelvetrano (Ibidem, 46).

${ }_{35}$ Così Di Castro, Avvertimenti, 60: «trattavansi le cause del genero dinanzi al suo suocero. Con che sincerità di giudici, chi sa quel, che sono giudici ordinarii, può immaginarselo». Tra queste va considerata la causa intentata da Pietro de Luna in merito alla successione di Maria Ventimiglia, moglie di Simone II, alle baronie di Ciminna e di Sperlinga: Orazio Cancila, I Ventimiglia di Geraci (1258-1619) (Palermo: Quaderni di Mediterranea-ricerche storiche, 2016), II, 304.

${ }^{36}$ Cancila, I Ventimiglia, 304. 
Vigliena $^{37}$. La loro figlia, Aloisia de Luna y Vega, sposerà Cesare Moncada, principe di Paternò, e si traferirà a Caltanissetta, dove sull'esempio della madre e in concorrenza con la matrigna Angela la Cerda, figlia del nuovo viceré Medinaceli - che intanto il padre rimasto vedovo aveva sposato nel 1563 - introdusse uno stile raffinato: ne fece una corte in tutti i sensi, luogo di incontro di musici, orafi, argentieri, letterati e pittori, dove persino il viceré Maqueda nel 1599 poté godere di una lussuosa ospitalità. A fine Cinquecento si deve proprio ad Aloisia la costruzione di un immenso complesso patrimoniale: sposò in seconde nozze il duca di Montalto Antonio Aragona, a sua volta vedovo di Maria la Cerda (altra figlia del Medinaceli), e organizzò il matrimonio tra i loro due figli Francesco II Moncada e la duchessa Maria Aragona Cardona la Cerda, nati dalle precedenti unioni, aggregando in tal modo due delle maggiori casate siciliane e ponendo così le basi della potenza e del prestigio dei Moncada nel Seicento ${ }^{38}$. Nel giro di tre generazioni il salto da Caltanissetta a Madrid proiettò la famiglia ai vertici dell'aristocrazia castigliana ${ }^{39}$.

Un altro matrimonio celebre fu quello tra Anna Maria Pacheco e Mendoza, nipote (figlia del fratello) del viceré Juan Fernández Pacheco, marchese di Villena, e Placido Fardella, il giovinetto marchese di San Lorenzo Xitta. L'unione consentì ai Fardella l'ingresso nel livello più elevato della gerarchia nobiliare siciliana. Il contratto matrimoniale fu stipulato nel 1607 dallo stesso viceré nella sua qualità di tutore di Maria, cui venne assicurata una cospicua dote e la promessa al futuro sposo dell'intercessione presso Filippo III per l'acquisizione del titolo di principe o duca. La successiva concessione vicereale della licentia populandi, che diede origine a Paceco, rappresentò per Placido una tappa importante verso l'acquisizione del titolo di principe, ma anche la possibilità di disporre di una sede adeguata al nuovo rango, che invece il piccolo borgo di San Lorenzo non consentiva. Il peso acquisito dai Fardella, pur tra qualche iniziale contrapposizione, comportò a Trapani il controllo politico dell'università, in particolare del procedimento di costituzione delle mastre e dell'elezione dei giurati, facendone gli interlocutori privilegiati del viceré Villena nelle questioni della città ${ }^{40}$.

A mettere in guardia il viceré dall'incoraggiare invece i matrimoni tra criados del suo seguito e donne siciliane - che «pensano col favore del Palazzo» di migliorare la propria posizione e influenza sociale - ci pensò Scipione di Castro nei suoi Avvertimenti al viceré Colonna. Era nota la grande disponibilità dei palermitani, soprattutto, nei confronti dei forasteros e la propensione a concedere le proprie figlie in matrimonio agli

${ }^{37}$ Antonino Marrone, Bivona città feudale (Caltanissetta-Roma: Sciascia editore, 1987), t. I, 152-153.

38 Per la ricostruzione puntuale della vicenda, cfr. Lina Scalisi e Rita Loredana Foti, "Il governo dei Moncada (1567-1672)", in La Sicilia dei Moncada. Le corti, l'arte e la cultura nei secoli XVI-XVII, a cura di Lina Scalisi (Catania: Domenico Sanfilippo Editore, 2006), 23-25; ma anche Domenico Ligresti, "Le piccole corti aristocratiche nella Sicilia spagnola", Archivio Storico per la Sicilia orientale 94/1 (1998): 11-35. In sintesi, Medinaceli sistemò Maria con Antonio Aragona, duca di Montalto; Angela con Pietro de Luna in seconde nozze; Bianca con Ferdinando de Silva, conte de Cifuentes.

39 Pilo, Luigi Guglielmo Moncada, 31-35.

${ }^{40}$ Sui Fardella e la fondazione di Paceco, cfr. Francesco Benigno, Una casa, una terra. Ricerche su Paceco, paese nuovo nella Sicilia del Sei e Settecento (Catania: CUECM, 1985). 
spagnoli. Simbolo della città era d'altra parte il Genio, un vecchio dalla barba lunga con una corona in testa e in braccio un serpente che gli succhiava il petto, ai piedi del quale campeggiava su un cesto pieno d'oro la scritta Panhormus conca aurea suos devorat, alienos nutrit ${ }^{41}$. Di Castro era però convinto che tali unioni fossero fonti «di nimicitie immortali», che inevitabilmente coinvolgevano il viceré, macchiandone suo malgrado la reputazione, perché esse «cominciaranno dal privato, et finiranno nel publico» ${ }^{42}$. Dinanzi a un contenzioso giudiziario i giudici biennali erano infatti inclini a favorire le «persone di palazzo», nella speranza di riceverne qualche incarico, al quale «non sarebbero pervenuti giammai per li loro meritis ${ }^{43}$. Così egli elenca una serie di casi di liti «rabbiose», che coinvolsero ben cinque cortigiani «principali» del viceré duca di Medinaceli, tanto che «la sua casa pareva un inferno», e gli procurarono inimicizie come quelle del barone di Cerami, del conte di San Marco o del potente duca di Terranova. Un'attività quella del viceré che procedeva tra equilibrismi continui e richiedeva notevole abilità di destreggiarsi tra interessi contrapposti. Ciò che poteva anche ridisegnare alleanze o generare nuove ostilità.

Anche il marchese di Pescara si trovò implicato in una lite tra l'inquisitore Giovanni Bezerra e un gentiluomo, suocero del suo cameriere ${ }^{44}$. In verità la storiografia ha appurato come le ragioni del contrasto con l'inquisitore fossero ben altre, prevalentemente legate alla pubblicazione nel 1570 di un capitolo che sottraeva al foro del Santo Uffizio i suoi ufficiali e familiari colpevoli di certi reati comuni; ma anche all'atteggiamento ostile dell'inquisitore nei confronti dei gesuiti, e al suo evidente appoggio alla fazione baronale che si opponeva alla riforma dei Tribunali, tanto da portare il viceré ad affermare che «Bezerra et lui non potevano stare in un medesimo Regno» ${ }^{45}$.

Il marchese di Pescara si era invece opposto al «maneggio» di due matrimoni tanto desiderati e raccomandati dal potente Ruy Gómez de Silva, principe di Eboli, quelli del nipote don Diego de Silva e del cugino Fernando de Silva, conte di Cifuentes. Quest'ultimo in particolare aveva messo gli occhi sulla giovane marchesa di Militello Dorotea Barresi e Santapau, ricchissima ereditiera grazie alla vedovanza seguita ai suoi precedenti matrimoni nel 1550 con Giovanni Branciforte, conte di Mazzarino, e nel 1567 con Vincenzo Barresi, marchese di Militello. Cifuentes finì invece con lo sposare Bianca, una delle figlie del viceré la Cerda, duca di Medinaceli; Dorotea alla fine, nel 1572, sposò in terze nozze Juan de Zuñiga y Requisens, personaggio di grande rilievo, ambasciatore a Roma presso la Santa Sede e poi viceré di Napoli ${ }^{46}$. Trascorse alcuni anni alla corte di Madrid dove fu nominata governatrice della casa dell'infante Filippo III, collocazione strategica per migliorare le sorti del proprio casato. Il figlio di primo

\footnotetext{
${ }^{41}$ Cisneros, Relación, 12; Pietro Corsetto, "Instruccion para el principe Filiberto quando fue al Virreynato de Sicilia", in Il governo della Sicilia in due Relazioni del primo Seicento, edizione a cura di Vittorio Sciuti Russi (Napoli: Jovene, 1984), 89.

42 Di Castro, Avvertimenti, 76.

${ }^{43}$ Ibidem, 75.

44 Ibidem, 48-49.

${ }^{45}$ Ibidem, 49.

46 Scalisi, "Magnus Siculus", 189.
} 
letto Fabrizio Branciforte, principe di Butera, che aveva accumulato un imponente dissesto finanziario per circa 200.000 scudi, riuscì così in parte a colmarlo grazie agli accordi matrimoniali fra il proprio figlio Francesco e Giovanna d'Austria, figlia di don Juan de Austria, il fratellastro di Filippo $\mathrm{II}^{47}$. Decisivo era stato l'interessamento al caso del viceré Lorenzo Suarez de Figueroa duca di Feria, grazie alla cui mediazione Filippo III era stato particolarmente generoso col principe Fabrizio, che si era assicurato una cospicua rendita personale di 10.000 scudi annui, scaricando sulla dote (60.000 mila scudi oltre ad argenteria, gioielli e corredo) della nuora buona parte dei debiti che gravavano anche sul figlio. Ne seguì un'annosa lite tra i due, con la denuncia al Consiglio d'Italia del comportamento scorretto del suocero da parte di Giovanna. Una lunga vicenda nella quale seppure in tempi diversi anche i viceré Villena e De Castro furono in qualche modo coinvolti, mediando tra l'interesse a guadagnarsi il favore del potente principe di Butera, primo titolo del regno, e l'attenzione nei confronti di una principessa di sangue reale.

Sono soltanto alcuni casi particolari, che concorrono a delineare il quadro in cui le élites siciliane entrarono in gioco, cogliendo opportunità, sfruttando situazioni, ma anche subendo mosse e contromosse di un gioco più ampio, spesso sovranazionale, che non sempre furono capaci di controllare e dirigere come volevano. D'altra parte, solamente appoggi, relazioni e alleanze preparate con cura, anche nel corso di diverse generazioni, consentirono ad alcune personalità di potersi proiettare su scenari altrimenti irraggiungibili, in «un complesso universo di fitti contatti informali attraverso cui passavano relazioni politiche di notevole importanza» ${ }^{48}$ : legami privati che segnavano però alleanze politiche e influenzavano scelte rilevanti per la vita pubblica.

\section{L'APPARATO DI PALAZZO: AULICI E UFFICIALI DI CAMERA}

Addetti alla persona del viceré erano una serie di figure sul modello delle altre corti europee, seppure in Sicilia in scala ridotta. Di «aulici e ufficiali di camera» si occupa un volumetto del marchese di Villabianca del XVIII secolo, che ci consente seppure in sintesi - e talvolta in una proiezione più settecentesca - di delineare le mansioni di una sorta di nobiltà di servizio, che assisteva il viceré sia limitatamente a una sfera di ambito più domestico sia in riferimento a ruoli che avevano una valenza più propriamente pubblica ${ }^{49}$. Si tratta in ogni caso di figure che, per quanto minori, avevano un accesso diretto alla sua persona e potevano istaurare con lui un rapporto di confidenza e di intimità piuttosto elevato. Così non sorprende che si trattasse

${ }^{47}$ Nicoletta Bazzano, "Pietraperzia Branciforte Barresi, Francesco", Diæionario Biografico degli Italiani, vol. 83 (2015), online: https://www.treccani.it/enciclopedia/pietraperziabrancifortebarresifrancesco ( Dizionario-Biografico)/ (ultima consulta: 08/11/2021).

${ }^{48}$ Cfr. Francesco Benigno, "A patti con la monarchia degli Asburgo? La Sicilia spagnola tra integrazione e conflitto", in Studi storici dedicati a Orazio Cancila, a cura di Antonino Giuffrida, Fabrizio D’Avenia e Daniele Palermo (Palermo: Quaderni di Mediterranea-ricerche storiche, 2011), vol. I, $373-$ 392.

${ }^{49} \mathrm{BCPa}$, ms. Qq E 109: Francesco Maria Emanuele Gaetani marchese di Villabianca, Degli Aulici e ufficiali di camera de' viceré di Sicilia, in Opuscoli palermitani, t. XXX. 
comunque di posizioni ambite proprio per la vicinanza fisica, che conferiva loro un'aurea di onore e di privilegio.

Il Cameriere maggiore vestiva e spogliava il viceré, gli cingeva la spada e gli porgeva guanti e cappello. Di fatto la sua mansione servile era ampiamente ricompensata dalla prossimità al principe e dall'alto onorario che gli era corrisposto, tale da consentire a diversi di acquisire titoli e feudi, realizzando un notevole salto sociale. I parlamenti, ad esempio, offrivano spesso un donativo di ben 200 onze a favore del cameriere contro le 60 per protonotaro, luogotenente e segretari; e le 40 per i portieri di camera, segno evidente del particolare ruolo che gli era riconosciuto. Scorrendo i nomi di coloro che ne svolsero il compito, emerge che per lo più erano spagnoli, personaggi evidentemente sui quali ciascun viceré riponeva grande fiducia ${ }^{50}$. Il cameriere inoltre disciplinava l'accesso alle stanze del viceré, quasi fosse una sorta di primo portiere di camera, un filtro cui era affidato un enorme potere discrezionale.

I gentiluomini di camera (maggiordomi) erano personaggi di nobile condizione che gli facevano «corte e corona sì nelle private che nelle grandi funzioni pubbliche, andandovi in carrozza» e precedendo alla testa dei cortei. Spesso erano reclutati tra $i$ quadri delle compagnie militari, come l'ordine dei Gerosolimitani o di San Giacomo, ma soprattutto nel Settecento facevano parte della classe senatoria di Palermo. Il cavallerizzo maggiore invece procedeva a cavallo a lato della carrozza vicereale e aveva la stessa dignità dei maggiordomi, sovrintendeva alle scuderie e ai cavallerizzi, anche se non si occupava del mantenimento e della cura delle carrozze. Una figura particolare era quella del governatore della Regia Razza, nominato dal viceré e selezionato tra membri del seguito vicereale, spesso dai ranghi più elevati dell'esercito, per lo più uno spagnolo $^{51}$. Era supportato da un vero e proprio apparato amministrativo, con ufficiali preposti alla contabilità e alla logistica (strumenti e attrezzature), ma anche personale più a diretto contatto con gli animali. Tra questi il cavalcatore, una sorta di maestro di equitazione, era preposto alla domatura e all'addestramento dei cavalli, ma anche alla selezione degli animali da riproduzione, nonché di quelli da destinare alla Corte o al mercato. Allevamento, riproduzione, cura dei cavalli erano snodi centrali anche del sistema difensivo e delle comunicazioni del regno. Il viceré de Vega, che - come si è detto - fu in prima linea nella militarizzazione del regno, non a caso si interessò sia alla

${ }^{50} \mathrm{BCPa}$, ms. Qq E 109: Francesco Maria Emanuele Gaetani marchese di Villabianca, Degli Aulici e ufficiali di camera de' viceré di Sicilia, in Opuscoli palermitani, t. XXX, ff. 20r-23v. Ecco alcuni nomi con una indicazione cronologica e il viceré di riferimento: Pietro Varrentoy (1554): viceré de Vega; Scipione Manganello (1570): viceré marchese di Pescara; Pietro de Landecha (1580): viceré Diego Enriquez de Guzmán; Ambrogio Calì (1570): presidente del regno marchese di Geraci, Giovanni III Ventimiglia; Gabriele Cardines (1600): viceré duca di Maqueda; Antonio Chiros (1636): presidente del regno Luigi Moncada duca di Montalto; Francesco Orioles, conte d'Alcantara (1638): presidente del regno Luigi Moncada duca di Montalto; Alessio del Hierico (1645): viceré Piero Faxardo, marchese de Los Velez; Giovanni Reira (1651): luogotenente del regno Antonio Brìcel Ronchiglio.

${ }^{51}$ Maurizio Vesco, "La Regia Razza di cavalli e le scuderie monumentali nella Sicilia degli Asburgo: il modello «negato» delle cavallerizze dei Palazzi Reali di Palermo e Messina", in Las caballerizas reales y el mundo d caballo, a cura di Juan Aranda Doncel e José Martínez Millán (Cordoba: Instituto Universitario "La Corte e Europa", 2016), 394-395. 
riproduzione sia alla commercializzazione dei cavalli, considerandoli un fattore assolutamente preminente per la sicurezza militare della Sicilia ${ }^{52}$.

Molto diverso era il ruolo del capitano della guardia alemanna, esponente della nobiltà di rango, figura di rilievo («principale e confidente») al quale era affidata la protezione fisica del viceré, che lo sceglieva a suo piacimento, ma piuttosto mal pagato (otto scudi al mese): era a capo di quaranta alabardieri alemanni a cavallo, retribuiti quattro scudi al mese per un totale di 2020 scudi (808 onze) l'anno ${ }^{53}$. Alcuni viceré disponevano anche di una guardia di venti (o ventidue) cavalli borgognoni leggeri, al soldo di sette scudi al mese per ciascuno, ma i più ne fecero a meno ritenendola troppo $\operatorname{costosa}^{54}$. Nel 1655 al servizio era impegnato Pietro Bonanno principe di Roccafiorita, appartenente a un casato di origine siracusana, legato nel passato all'ordine dei cavalieri gerosolimitani.

L'interesse per il mondo cavalleresco era preminente nella cultura dell'epoca, tuttavia non sempre collegato a un incarico militare effettivo. La presenza di siciliani al servizio del re non va sottovalutata ed è ampiamente documentata, anche se le carriere veramente significative non sono numerose, perché i vertici erano spesso scelti tra gli spagnoli. Ciò nonostante, a livello del Regno la nobiltà siciliana non mostrò disinteresse per le questioni militari, distinguendosi nella guerra sul mare e soprattutto nel controllo del territorio, nella milizia del Regno, o al comando di castelli e fortificazioni. I ruoli più prestigiosi di vicario del Regno o di vicario di Valle erano generalmente ricoperti da rappresentanti delle maggiori casate, personaggi esperti e affidabili, mentre quelli di capitano d'arme a guerra, limitati a singole città o aree minori, erano di solito attribuiti agli esponenti delle famiglie baronali e patrizie di medio livello ${ }^{55}$.

In questo contesto culturale di attenzione alla cultura del cavallo e al mondo cavalleresco il viceré Garcia Álvarez de Toledo, condottiero e uomo d'arme, si adoperò per la realizzazione di grandi cavallerizze nei due palazzi di Palermo e Messina, e promosse anche l'istituzione nel 1566 di una congregazione «intitulata La Cavallaria», l'Accademia dei Cavalieri, della quale entrò a far parte col figlio Pedro, cooptandovi elementi della maggiore nobiltà del Regno ${ }^{56}$. A seguire ne sorsero altre: a Messina l'Accademia della Stella (1595), a Catania l'Accademia dei Chiari, a Siracusa l'Accademia dei Cavalieri della fede, associazioni nobiliari che divennero anche spazi

${ }^{52}$ Giuffrida, "La fortezza indifesa", 254.

${ }^{53} \mathrm{BCPa}$, Qq C 22: Relazione del governo di Sicilia, c. 13r. I dati dello stipendio si trovano in Alfonso Crivella, Trattato di Sicilia (1593), a cura di Adelaide Baviera Albanese (Caltanissetta-Roma: Sciascia editore, 1970), 112.

${ }^{54}$ Cisneros, Relación, 37. Cfr. anche Josefina Mateu Ibars, “«Noticias del Reyno de Sicilia y govierno para los virreys». Manuscrito de la Biblioteca Comunale de Palermo”, Boletín de la Real Academia de Buenas Letras de Barcelona 30 (1963-64): 190 (si riferisce al ms. Qq E 63).

${ }^{55}$ Domenico Ligresti, Le armi dei Siciliani Cavalleria, guerra e moneta nella Sicilia spagnola (secoli XV-XVII) (Palermo: Ebook Mediterranea-ricerche storiche, 2013), che offre un panorama articolato sulla questione.

56 Vesco, "La Regia Razza di cavalli", 400-405. Sull’Accademia dei Cavalieri, cfr. Salvatore Salomone-Marino, "La congregazione dei Cavalieri d'armi e le pubbliche giostre in Palermo nel secolo XVI. Notizie e documenti", Nuove effemeridi siciliane - serie III 5 (1877): 103-139. 
in cui rappresentare le proprie virtù eroiche, in quanto curavano l'ideazione e l'organizzazione di spettacoli e cerimonie e promuovevano l'addestramento dei giovani cavalieri. Era un mondo che comunque nel corso del XVII secolo assumeva sempre più una connotazione simbolica, in cui formazione e abilità militari facevano ormai da sfondo a giostre, tornei, giochi di guerra: danza, poesia, scherma, caccia e addestramento dei cavalli, duello ne erano ormai i principali aspetti. Le Accademie in genere, militari e letterarie, ebbero comunque anche un'importante funzione non solo in relazione alla socialità aristocratica, ma anche come luogo di affiliazione o di creazione di reti e legami personali ${ }^{57}$.

Su un livello più ufficiale e meno domestico si collocavano i quattro portieri di camera, che dipendevano dal Protonotaro. Svolgevano inoltre il ruolo di portieri della Regia corte in occasione dei parlamenti. Erano ancora i portieri che riferivano ai Tribunali alcuni ordini “orali” («a boca») del viceré, compito questo considerato «de mucha confianza» ${ }^{58}$. Introducevano i feudatari di nuova nomina quando ricevevano l'investitura e giuravano fedeltà, e venivano indicati perciò nell'atto come testimoni della funzione, prendevano l'uno per cento sulle rendite feudali, calcolate comunque su dati per lo più risalenti nel tempo e non aggiornati. Più in generale presenziavano $\mathrm{a}$ tutte le cerimonie di conferimento degli uffici di governo, ricevendo un contributo sugli atti di possesso secondo un tariffario ben stabilito. D'altra parte, il loro ufficio era vendibile e vitalizio, e pertanto la corresponsione di queste somme valeva a pagamento di un servizio offerto, come da prassi. Nel Parlamento del 1615, sotto il viceré Osuna, furono concessi ai portieri compensi in denaro piuttosto cospicui seppure una tantum, addirittura 200 onze, di cui 80 soltanto per i servizi offerti durante la seduta ad Antonio Albigiano, il cui nome risulta successivamente dal 1620-1622 come segretario del regno ${ }^{59}$. Essi avevano inoltre il compito di presidiare le porte della camera in cui il viceré teneva la sua udienza, annunciando ad alta voce il nome di colui che doveva essere ammesso al suo cospetto. La funzione era istituzionale e presupponeva la conoscenza del cerimoniale, in quanto l'ingresso era regolato da una successione ben definita: prima i titolati, poi i ministri regi e i consiglieri, di seguito i nobili e infine tutti gli altri. Ma non pochi, soprattutto ministri e togati, cercavano di introdursi saltando le precedenze.

La settimana del viceré era scandita da una serie di appuntamenti secondo una precisa calendarizzazione, che prevedeva oltre agli incontri istituzionali anche udienze pubbliche e private. Nella sua relazione il giurista Francesco Fortunato lo avvertiva dell'opportunità di tenere il Palazzo aperto a tutti coloro che chiedessero di incontrarlo, ricevendoli pubblicamente e frequentemente. Occorreva - suggeriva - trattare con familiarità i nobili, ma anche gli esponenti della classe dirigente, consiglieri e ministri che manovrano le leve del potere e col viceré esercitano l'attività di governo, riservando

\footnotetext{
${ }^{57}$ Bazzano, Palermo fastosissima, 54-56.

${ }^{58}$ Cisneros, Relación, 28.

${ }^{59}$ Il Parlamento del 1615. Atti e documenti, edizione a cura di Francesco Vergara (Catania: Bonanno, 1991), 21.
} 
loro una anticamera conveniente ${ }^{60}$. Indubbiamente questo era un momento importante dell'attività viceregia, scandito sempre dal cerimoniale col suo linguaggio e la sua cultura. A detta dei messinesi a Palermo si mandavano «le più principali dame a corteggiare le signore vicereggine» e i viceré erano «circondati da moltitudine d'interressati adulatori», che «facendosi cogniti in palazzo con l'assiduità, impetrano facilmente le dilazioni de' loro debiti, ed insieme gli ufici, che donano i signori viceré», provocando invece un "gravissimo danno» al regno. Ma «il continuo corteggio, che si fa in Palermo il dì e la notte, rubba inutilmente il tempo, che doverebbe di ragione impiegarsi alla spedizione delle cause dei negozianti» ${ }^{61}$.

\section{IL SEGRETARIO DEL VICERÉ}

Tra i criados del viceré «el mas privado» era il segretario particolare, che secondo il giurista Francesco Fortunato (1591) ricopriva un incarico «de señor particular y no es oficial publico del Reyno». ${ }^{62}$ Dello stesso avviso era Pietro Corsetto che nella sua Relazione (1621) raccomandava che il viceré lo considerasse «mas por ministro áulico que público, y por esto no deve ocuparle en negocios» ${ }^{63}$. Questa figura non va infatti confusa con i Segretari del regno, ritenuti i continuatori degli antichi Referendari, che si inquadravano nel contesto istituzionale: fungevano da tramite tra il viceré e i privati e tra il viceré e le magistrature, ed erano pertanto in stretto collegamento col Protonotaro del regno ${ }^{64}$. Di fatto dalle loro mani passavano tutti gli affari che riguardavano l'amministrazione del regno. Ma non sorprende che talvolta entrassero in contrasto con il segretario personale del vicerée ${ }^{65}$.

Questi operava al di fuori della struttura istituzionale, su un piano meno amministrativo e più legato alla sfera informale, anche politica, e confidenziale. L'ufficio era molto remunerativo, se i proventi «lícitos» - quasi a voler fare intendere che ce ne fossero altri anche illeciti - ammontavano a mille scudi l'anno, includendovi anche i duecento del salario ${ }^{66}$. A lui, uomo di sua fiducia e del suo seguito, il viceré avrebbe dovuto affidare solo il disbrigo della corrispondenza estera (lettere di cortesia) e riservata col sovrano, e anche alcune questioni che afferivano alla sfera militare, come

\footnotetext{
${ }^{60} \mathrm{Si}$ vedano - ma il tema è ricorrente - anche i consigli contenuti nel capito XLVII Acuerdos para el Virrey açerca de su govierno, della Relazione del 1655: Mateu Ibars, "«Noticias del Reyno de Sicilia”: 226233.

${ }^{61}$ Placido Reina (Idoplare Copa), L’Idra dicapitata (Vicenza: per Gio. Antonio Gabucci, 1662), 44.

${ }^{62}$ Los avertimientos del doctor Fortunato sobre el govierno de Sicilia (1591), edizione a cura di Adelaide Baviera Albanese (Palermo: Società Siciliana per la Storia Patria, 1976), 83-84.

${ }^{63}$ Corsetto, "Instruccion", 100.

64 Adelaide Baviera Albanese, Diritto pubblico e istiturioni amministrative in Sicilia. Le fonti (Roma: Il Centro di ricerca, 1981), 102.

${ }^{65}$ Nell'area milanese, si veda Gianluigi Barni, "Dissensi fra i segretari del Gran Cancelliere e i segretari del Governatore a Milano", Archivio Storico Lombardo 40 (1933): 516-527. Sulla segreteria del gran cancelliere a Milano, cfr. Gianvittorio Signorotto, Milano spagnola. Guerra, istituzioni, uomini di governo (1635-1660) (Firenze: Sansoni, 1996), 104-105; anche Cinzia Cremonini, Il Consiglio Segreto tra "interim" $e$ prassi quotidiana (1622-1706), in La Lombardia spagnola, 239-241.

${ }^{66}$ Cisneros, Relación, 27.
} 
la concessione di patenti e brevetti o la sistemazione degli alloggiamenti militari. Del suo segretario Iohan del Rio, il viceré Moncada esaltava la grande capacità di lavoro «et di notte et di iorno, fatigando in lo scriviri et copiari letteri, instruttioni, capituli et altri scripturi che da nui continuamenti su trasmisi a Sua Maestati et in scriviri a multi altri potentati et di Sua Altezza ambaxiaturi per diversi parti di lo mundo» ${ }^{67}$.

La loro influenza era però negli anni talmente cresciuta da sconfinare nell'abuso. D'altra parte, il segretario apriva lettere e dispacci e ne riferiva al viceré, cosicché veniva per primo a conoscenza di decisioni e ordini da Madrid; sottoponeva inoltre alla firma del viceré la documentazione senza che in molti casi questo ne facesse una lettura preventiva. Tra l'altro era noto che talvolta i viceré gli affidavano l'elezione degli ufficiali minori, che spettava invece al Protonotaro, cosa che «ha salido muy horrada» ${ }^{68}$. Il giurista Fortunato - a difesa delle prerogative del regno - sottolineava l'opportunità che fossero gli organi istituzionalmente preposti a occuparsi delle diverse attività amministrative e giurisdizionali e nei suoi Avertimientos ricordava come al tempo del viceré Diego Enríquez y Guzmán, conte di Alba de Liste (Alvadeliste) (1585-1592) erano sorti «muchos inconvenientes en muchos negocios» a causa dell'invadenza del suo segretario, che voleva «abrazarlos todos y aplicarlos à si» ${ }^{69}$. E ironicamente insinuava anche che le lettere che il marchese di Pescara scriveva al sovrano «parecer que eran mas del secretario [Juan Francisco Locadelo] que suyas ${ }^{70}$.

Nella Relazione del dottor Bernardino Masbel (1694) dedicata al Senato di Palermo si fa riferimento a una segreteria di Stato e Guerra a disposizione del vicerée ${ }^{71}$, probabilmente istituita a seguito del permesso accordato da Filippo III di tenere una propria segreteria di governo ${ }^{72}$. Essa era composta dal suo segretario, da un ufficiale maggiore e da altri inferiori, tutti spagnoli e «degni di tal carica». Al suo interno un ruolo particolarmente sensibile era svolto dall" archivaro che «delli papelli e registri di essa per esser di molta importanza ha cura»: «spagnolo di gran confidenza», il quale senza decreto particolare del viceré non avrebbe potuto trasmettere copie delle scritture conservate nel suo archivio ${ }^{73}$. Il ruolo del segretario si andava così sempre più strutturando in maniera formale, non senza però generare ulteriore conflittualità all'interno della compagine amministrativa. Filippo III nel 1610 invitava il marchese di Vigliena (1606-1610) a porre un freno all'eccesso e disordine «con que los secretarios de algunos virreyes vestros antecessores han puesto mano così en toda manera de negocios», usurpando le mansioni di altri ufficiali ${ }^{74}$. Ancora nelle sue istruzioni ad Albuquerque (1627) Filippo IV gli ricordava espressamente «che non abbiano altro

${ }^{67}$ ASPa, Real Cancelleria, reg. 227 (a. 1508), c. 77, cit. in Baviera Albanese, Diritto pubblico, 95.

${ }^{68}$ Corsetto, "Instruccion", 100.

${ }^{69}$ Los advertimientos, 84 . Il tema ricorre anche in altre successive relazioni.

70 Ibidem.

${ }^{71}$ Bernardino Masbel, Descrizione e relatione del governo di stato e guerra del regno di Sicilia (Palermo: Pietro Coppola, 1694), 48.

${ }^{72}$ Giardina, L'istituto del Viceré, 280. L'archivio della Real Segreteria di Sicilia comincia con l'anno 1611, anno in cui fu esecutoriata una riforma dell'istituto: si deve al viceré Osuna l'organizzazione e la regolare tenuta dell'archivio (Baviera Albanese, Diritto pubblico, 97).

${ }^{73}$ Masbel, Descrizione, 48.

${ }^{74}$ Giardina, L'istituto del Viceré, 281. 
ufficio, se non scrivere lettere, perché questo veramente gli tocca, et è concernente a quest'ufficio», e di non permettere eccezioni spesso causa di «gravi inconvenienti» ${ }^{75}$. Ordinava inoltre che non chiedessero emolumenti oltre il dovuto per servizi prestati (ad esempio sui passaporti e licenze dei soldati) e che sulla base di loro relazioni non si facesse «nessuna grazia di delitti, di contumacie, plegerie» né si assumesse alcun provvedimento di giustizia o finanziario. Esortava più tardi anche il duca di Alcalà (1632-1636) a non adoperare la propria segreteria per affari spettanti ad altre magistrature, ma tali abusi furono ampiamente proseguiti, soprattutto sotto il conte di Santo Stefano (1679-1687) e il successore duca di Uzeda (1687-1696) ${ }^{76}$.

Accusati spesso di corruzione, di agire con arroganza, di operare dispoticamente, tra i casi più noti possono segnalarsi quello di Giovanni Mahona, segretario di Ferrante Gonzaga, che seguì anche a Milano, di cui Scipione di Castro offre un ritratto nefasto, descrivendolo come «huomo rapacissiomo et risoluto ad ogni scelleratezza per qual si voglia mediocre interesse», rilevando che a Milano fece «mercantia della giustitia, come soleva in Sicilia», sino a vendere grazie ed uffici «a suo modo» ${ }^{77}$. Ma si consideri anche Pedro de Cisneros, il fidato segretario di Marco Antonio Colonna, poi arrestato e condannato alla pena capitale per ordine dello stesso viceré (1585) probabilmente per dimostrare la propria inflessibilità e imparzialità, con l'accusa di essere stato responsabile di «molte frodi e in particolare di una certa esportazione di denaro confessata da lui stesso, e oltre a questo di molte estorsioni a persone di origine spagnola» ${ }^{78}$. Al centro di uno scontro tra il viceré e il visitatore Gregorio Bravo, Cisneros inizialmente accettò di parlare con il visitatore, che voleva servirsene per incastrare Colonna, ma poi per salvarsi la vita, pur sotto tortura, ritrattò, accusando Bravo di avergli estorto le confessioni. Commutata la pena in relegazione per dieci anni, si dedicò alla redazione della Relación, destinandola al nuovo viceré conte di Alba de Liste, da cui sperava ulteriori atti di clemenza. Pessima fama ebbe anche Juan Francisco Locadelo, ritenuto dal marchese di Pescara «per intelligente, sincero et destro nel maneggiar de' negotii», ma rivelatosi invece «ignorante, arrogante, incivile», insomma «il primo intoppo» sul percorso del viceré, cui mentre visse «fece bere molti calici ben amaris ${ }^{79}$. Litigiosissimo, si intromise pesantemente nella gestione degli uffici del regno, scontrandosi aspramente col Conservatore del Real Patrimonio Pedro Velazquez «uomo avuto dal Re in opinion di sincero et capace di governo», il quale recatosi a corte, con l'appoggio del consultore Andrea Arduino, scalfi la fiducia di cui il viceré godeva presso il sovrano.

Giudizio assai negativo è anche quello nei confronti del segretario del viceré Juan Francisco Pacheco duca d'Uzeda (1687): alla morte del fidato Felice Lucio Spinosa, considerato uomo probo, subentrò Feliz Cruz Haedo «uomo in verità di grandi talenti, ma furbo e astuto; il quale da destro cortigiano, consigliando gli studi

${ }^{75}$ Istruqioni date al viceré duca di Albucherche, cap. 106, cc. 37r-v (Madrid 30 maggio 1627).

${ }^{76}$ Giardina, L'istituto del Viceré, 281.

${ }^{77}$ Cit. in Saitta, Note, 86.

${ }^{78}$ Cit. in Bazzano, Marco Antonio Colonna, 292. Sul rapporto tra il visitatore e il viceré cfr. Ibidem, 291-297, ma anche Vittorio Sciuti Russi, Nota al testo, en Cisneros, Relación, XIII-XXXIV.

${ }^{79}$ Di Castro, Avvertimenti, 47-48; Cisneros, Relación, 50-51; Zapperi, "Avalos". 
amati al viceré, prese a suo carico tutta la cura del Governo; e arrogandosi ogni autorità, cominciò ad operare dispoticamente, spogliando i Tribunali, e i Ministri della loro autorità, vendendo le cariche e condannando i rei a multe pecuniarie, con le quali si arricchiva, e lasciava intanto, che impunemente si commettessero i delitti da coloro, che erano ricchi e in stato di comprare la dovuta pena ${ }^{80}$. Una valutazione pesante su un personaggio, che potenziò enormemente il ruolo della segreteria del viceré, condizionandone fortemente l'attività di governo. Il duca d'Uzeda fu ricordato in effetti per la sua ricchissima collezione di dipinti, sculture e antichità greco-romane, ma anche per la sua pregiatissima biblioteca privata con edizioni rare e antichi manoscritti, alcuni dei quali incamerati dopo la rivolta di Messina; oltre che per le numerose cerimonie pubbliche che lo videro protagonista insieme con la moglie in una cornice barocca di magnificenza e splendore ${ }^{81}$.

Tra i segretari non mancarono però esempi di uomini illustri, che si distinsero più per i loro interessi culturali che per gli intrighi politici. Francisco Ruiz de Castro, viceré di Sicilia negli anni 1616-1622, fu sempre supportato dal suo fidato segretario Juan de Lezcano, che lo seguì nella sua attività di ambasciatore a Venezia e a Roma, poi a Palermo come viceré: era un grande collezionista di dipinti italiani, che aveva acquistato soprattutto negli anni della sua permanenza a Roma tra il 1609 e il 1616, mentre pochissime sono le opere riconducibili ai suoi soggiorni in Sicilia o a Napoli ${ }^{82}$. Il viceré, che si segnalò come raffinato committente di opere d'arte, condivise con lui questi interessi. Promozione e diffusione della cultura rappresentarono ove presenti elementi di grande rilievo dell'attività dei viceré, spesso artefici di importanti interventi a livello urbanistico e architettonico, che negli anni mutarono il volto delle città grazie anche al coinvolgimento diretto e indiretto di privati ed enti ecclesiastici in una sorta di gara in cui ognuno autorappresentava il proprio onore e il proprio prestigio ${ }^{83}$.

${ }^{80}$ Di Blasi, Storia cronologica de' Vicerè, vol. III, 326. Sull'attività di Uzeda, cfr. anche Giuseppe Tricoli, Un periodo del governo spagnolo di Sicilia nella Relazione del viceré Uzeda (1687-1696) (Palermo: Edizioni Thule, 1980).

${ }^{81}$ Pablo Gonzáles Tornel, "Il duca d’Uzeda, la duchessa e l'apoteosi della festa barocca”, in La Sicilia dei viceré nell'età degli Asburgo, 248-255; Valeria Manfrè e Margarita Martín Velasco, "La corte virreinal siciliana del IV Duque de Uceda en Sicilia (1687-1696)", in En tierra de confluencias. Italia y la Monarquía de España, siglos XVI-XVIII, a cura di Cristina Bravo Lozano e Roberto Quirós Rosado (Valencia: Albatros Ediciones, 2013), 61-79.

82 Antonio Vannugli, "Il segretario Juan de Lezcano e la sua collezione di dipinti italiani”, in Centros de poder italianos en la monarquía hispánica (siglos XV-XVIII), a cura di José Martínez Millán e Manuel Rivero Rodríguez (Madrid: Polifemo, 2010), vol. III, 1487-1542.

${ }^{83}$ Sul rinnovamento urbano a Palermo e la dialettica tra poteri, cfr. Stefano Piazza, "Volontà governative e poteri locali nel rinnovamento urbano di Palermo", in Capitali senza re nella Monarchia spagnola. Identità, relazioni, immagini (secc. XVI-XVIII), a cura di Rossella Cancila (Palermo: Quaderni di Mediterranea-ricerche storiche, 2020), 151-163; Maurizio Vesco, "Un regno, due capitali. Opere pubbliche, politica dell'immagine e costruzione simbolica a Palermo e Messina (secc. XVI-XVII)", in Capitali senza re, 339-367. 


\section{IL LIVELLO ISTITUZIONALE: IL CONSIGLIO RISTRETTO}

Organo istituzionale consultivo era il Sacro Regio Consiglio, composto da un numero di membri oscillante tra i 26 e i 29 , tutti esponenti degli uffici più importanti del regno a stretto contatto con il vicerée ${ }^{84}$. Era presieduto da viceré e in sua assenza dal presidente della Gran Corte. Al suo interno tutti i componenti erano regnicoli, ad eccezione dello stesso viceré, del consultore e del Conservatore del Real patrimonio. Pietro Celestre nella sua Relazione del 1611 al viceré Osuna ne rileva tuttavia la perdita di importanza e l'incremento di autorità dei tre presidenti dei Tribunali - considerati $\mathrm{i}$ consiglieri naturali del viceré - e del consultore, con cui i viceré si consultavano preventivamente e che costituivano una sorta di consiglio ristretto (consessum Presidum et Consultoris). Era questa la giunta dei presidenti e consultore, che voluta da Colonna per controbilanciare l'elevato potere del consultore, acquisirà sempre più forza finendo col sostituire il Sacro Regio Consiglio, e divenendo parte integrante e indispensabile dell'ordinamento istituzionale siciliano, giacché si occupava delle questioni più spinose soprattutto in materia giuridica ${ }^{85}$.

Il consultore, estraneo all'ambiente locale, e dunque considerato formalmente libero da "parzialità", non era comunque un uomo del viceré, ma era scelto dal sovrano anche per controllarne l'operato e relazionargli per l'assunzione di provvedimenti che talora potevano essere spiacevolii ${ }^{86}$ I consultori erano perciò guardati con diffidenza e non solo dai viceré - per il loro rapporto diretto con la corte di Madrid. Da parte loro essi lamentavano spesso che i viceré li ignorassero e non richiedessero il loro parere come avrebbero invece dovuto fare; né mancarono di scontrarsi con il cerimoniale chiedendo posizionamenti più consoni al ruolo ricoperto ${ }^{87}$.

\footnotetext{
${ }^{84}$ Era formato dal consultore, dai tre presidenti dei Tribunali della Gran Corte, del Concistoro e del Real Patrimonio, dai sei maestri razionali, dai sei giudici della Gran Corte, dai tre giudici del Concistoro, dall'auditore generale, dal tesoriere, dal conservatore del Real Patrimonio, dal maestro portulano, dal protonotaro e dal maestro secreto. Cfr. Pietro Burgarella, "Verbali del Sacro Regio Consiglio di Sicilia del secolo XV", Archivio storico siciliano - serie IV 7 (1981): 115-210. Potrebbe assimilarsi al Consiglio segreto dello Stato di Milano: Cremonini, Il Consiglio Segreto, 225-261; ma anche Ugo Petronio, Il Senato di Milano. Istituzioni giuridiche ed esercizio del potere nel Ducato di Milano da Carlo V a Giuseppe II (Milano: Giuffrè, 1972), 140-141.

85 Adelaide Baviera Albanese, "L’ufficio del Consultore del viceré nel quadro delle riforme dell'amministrazione giudiziaria del sec. XVI in Sicilia", in Scritti minori, a cura di Adelaide Baviera Albanese (Soveria Mannelli: Rubbettino, 1993), 142. Sui compiti della Giunta cfr. Pietro Celestre, "Idea del Govierno del Reyno de Sicilia", in Il governo della Sicilia, 47-48; ma riferimenti si trovano pure in Corsetto, "Instruccion", 98, che ne parla come di una sorta di Consejo Colateral. Per il contesto politico in cui cominciò a operare, cfr. Bazzano, Marco Antonio Colonna, 299-300. La Baviera Albanese evidenzia però che l'archivio della Giunta ha inizio però solo nel 1690, cosa che le fa ipotizzare che la consacrazione giuridica dell'istituto sia avvenuta attorno a quegli anni.

86 Baviera Albanese, "L'ufficio del Consultore", 137-139.

${ }^{87}$ Ibidem, 142 (il caso del consultore Pietro de Léon che nel 1571 chiedeva di essere considerato come un quarto elemento del consiglio ristretto insieme con i tre presidenti); ibidem, 145 (Alonso Taboada Pardo nel 1578 chiedeva gli venisse concesso di sedere nei tribunali in uno speciale tronetto anziché in communi scanno).
} 
Andrea Arduino, scelto da Carlo V nel 1535 per affiancare Simone Ventimiglia, cui era stata affidata la presidenza del regno alla morte di Ettore Pignatelli, fu il primo nel regno. Rimarrà in carica per parecchi anni. In ottimi rapporti con Ferrante Gonzaga, fu considerato «ambiociosissimo» da Juan de la Cerda, duca di Medinaceli (1557-1565), che ne sperimentò il controllo serrato attraverso ben due visitas, che ebbero come esito la condanna dei consiglieri a lui più vicini e la revoca dello stesso prima della scadenza del triennio ${ }^{88}$. Ma anche col viceré de Vega (1547-1557) e col marchese di Pescara (1568-1571) l'Arduino entrò in urto. La sua posizione fu comunque solidissima, tanto che nel 1568 ricevette l'importantissima carica a vita di presidente del Tribunale del Real Patrimonio, con uno stipendio di ben 1000 scudi l'anno. D'altra parte, aveva proceduto a radicarsi nel regno, sposando nel 1542 una Gioeni, figlia del marchese di Castiglione.

Assai ambiguo è il ritratto che la storiografia ha disegnato del consultore al tempo del viceré Colonna, Alonso Taboada Pardo. Uomo dell'inquisitore generale Gaspar de Quiroga per conto del quale cercò di sostenere l'Inquisizione siciliana con cui il viceré era entrato in forte contrasto, divenne un punto di riferimento dell'opposizione che intanto si andava costruendo contro Colonna ${ }^{89}$. Tentò anche di ingerirsi in questioni che riguardano altre sfere istituzionali, provocando le rimostranze della Deputazione del regno, il cui scontro giurisdizionale con il consultore può essere però meglio inquadrato nel contesto più ampio della lotta politica che si sviluppa attorno alla condotta del viceré Colonna ${ }^{90}$. Taboada Pardo seppe inoltre fornire al visitatore generale Gregorio Bravo le informazioni che gli permisero di colpire i punti più fragili dell'amministrazione viceregia. Colonna reagì esautorando il ruolo del consultore con la creazione della giunta dei presidenti e consultore, in cui poteva contare dell'appoggio dei tre presidenti dei tre principali tribunali del regno; ma fu costretto anche a delle aperture che ne rendessero meno ostile l'attività in Sicilia in considerazione degli appoggi di cui il consultore godeva a Madrid.

Come il consultore, anche il conservatore del Real patrimonio era una figura istituzionalmente preminente, fra le più importanti nell'ordinamento istituzionale del regno di Sicilia, con compiti di controllo della legittimità giuridica degli atti concernenti la pubblica finanza e dell'operato degli ufficiali dell'amministrazione finanziaria e che comunque maneggiavano denaro pubblico ${ }^{91}$. Fortissimo fu lo scontro tra il viceré Colonna ed Esteván de Monreal, autore di un memoriale di assoluto discredito nei confronti del viceré e personaggio controverso che già era entrato in contrasto con $i$ predecessori del Colonna, sino ad essere imprigionato durante la presidenza del duca di Terranova ${ }^{92}$. Riabilitato, fu poi accusato dal viceré, che ne chiese al sovrano la rimozione, e sostituito da Miguel de Idiáquez.

${ }^{88}$ Sull'Arduino si veda Baviera Albanese, "L’ufficio del Consultore", 136-138; Roberto Zapperi, "Arduino, Andrea", Dizionario Biografico degli Italiani, vol. 4 (1962),online: https://www.treccani.it/encic lopedia/andrea-arduino (Dizionario-Biografico)/ (ultima consulta: 08/11/2021).

${ }^{89}$ Cfr. l'efficace ritratto del consultore Taboada Pardo in Bazzano, Marco Antonio Colonna, 270-272.

${ }^{90}$ Ibidem, 271.

${ }^{91}$ Baviera Albanese, "L’ufficio del Consultore", 18.

92 Bazzano, Marco Antonio Colonna, 266-267. 
In questo panorama di aspra lotta politica particolarmente rilevante era - come già accennato - il ruolo dei presidenti dei tre Tribunali (Gran Corte, Concistoro e Patrimonio): a loro erano affidate già dalla fine del Cinquecento importanti funzioni giurisdizionali, ma anche compiti fondamentali, come quello di proporre per ogni "piazza" le terne per le nomine dei giudici biennali della Gran Corte e del Concistoro, poi trasmesse dal viceré con il suo parere al Consiglio d'Italia. Risulta evidente il ruolo politico esercitato dai presidenti e l'enorme influenza che essi potevano vantare sui giudici biennali, le cui fortune e carriere dipendevano dal loro favore ${ }^{93}$. Si tratta di un assetto istituzionale che era emerso a seguito della riforma dei Tribunali varata in Sicilia da Filippo II nel 1569: una riforma delle magistrature di chiaro impianto antinobiliare, a vantaggio del ceto togato, che si assicurava le tre presidenze dei Tribunali (carica perpetua). Occorre però sottolineare che le aspirazioni di ascesa sociale dei togati, che puntavano alla terra e al titolo, e il sistema della biennalità delle cariche (perpetui erano solamente i sei maestri razionali del Patrimonio), che consolidava il rapporto di clientelismo tra ministero togato e baronaggio, finirono nella realtà per svuotare gli aspetti antibaronali della riforma voluta da Filippo II. Nel corso del Seicento «hombres de negocios», nobili, giuristi si avvantaggiarono delle massicce alienazioni di patrimonio reale, partecipando attivamente al mercato degli uffici e dei titoli, e utilizzando lo stato come fonte di profitto: ne furono coinvolti oltre a singoli personaggi, anche gruppi di potere interessati direttamente a speculazioni e affari, che con spregiudicatezza e arroganza riuscirono a controllare posizioni di grande rilievo istituzionale in un quadro ormai di complessiva debolezza istituzionale.

\section{IL PALAZZO DEL VICERÉ E LA QUESTIONE DELLA RESIDENZA: I RISCHI DI UNA CORTE ITINERANTE}

A sottolineare l'importanza che i Tribunali avevano assunto nella gestione del regno, è certo significativo che proprio negli anni Novanta del Cinquecento il viceré Maqueda (1598-1602) decidesse di trasferirli nella propria residenza, disponendone gli uffici attorno a un grande cortile a quadriportici su tre ordini, ai quali si accedeva attraverso un monumentale scalone ${ }^{94}$. A lui si deve inoltre la costruzione di una grande Sala o galleria, spazio di rappresentanza, di feste e rappresentazioni, ma anche luogo delle udienze pubbliche regolate da un rigido cerimoniale. Anche le sedute del Parlamento siciliano cominciarono a tenersi stabilmente all'interno del Palazzo reale. Le spese sostenute furono ingenti, ampiamente superati i 20.000 scudi annui stanziati per i lavori ${ }^{95}$, secondo quanto stabilito a partire dal Parlamento del 1570, che aveva fissato un donativo dei palazzi per quell'ammontare da ripartire in tre anni, proprio allo scopo di ristrutturare e migliorare le dimore reali.

\footnotetext{
${ }^{93}$ Su questi aspetti, cfr. Vittorio Sciuti Russi, Astrea in Sicilia (Napoli: Jovene, 1983), 94-97.

${ }^{94}$ Carlos Gonzáles Reyes, "Il governo di don Bernardino de Cárdenas, III duca di Maqueda, nella Sicilia di fine Cinquecento: potere e architettura", in La Sicilia dei viceré nell'età degli Asburgo, 177-181.

95 Ibidem, 180.
} 
Sempre più il Palazzo diveniva il centro di direzione politica e amministrativa del Regno, il luogo istituzionale per eccellenza, sede dei Tribunali e delle adunanze del Parlamento siciliano e non più solamente la residenza privata del viceré. In questo quadro rientra il trasferimento già nel 1553 per volontà di Juan de Vega della sede vicereale palermitana dal Castellammare al Palazzo reale dei Normanni, allora sede del tribunale dell'Inquisizione. È evidente che la riappropriazione dell'antica dimora dei re normanni aveva un significato ideologicamente rilevante, oltre che dalla forte valenza politica, come elemento di legittimazione della nuova monarchia sulla Sicilia in virtù della continuità dinastica con l'antica dinastia che aveva fondato la monarchia in Sicilia. Da allora continui furono gli interventi sia sulla facciata sia sugli ambienti interni e ogni viceré volle lasciarvi la propria impronta ${ }^{96}$.

Il fatto che i Tribunali e i loro archivi avessero sede nel Palazzo costituì uno degli elementi su cui si fondò la resistenza palermitana al trasferimento della corte a Messina ogni diciotto mesi secondo quanto stabilito dal controverso privilegio del 1591. È noto che le due città si contendessero la posizione di capitale del regno e ormai è dimostrata la centralità nella questione proprio della residenza dei Tribunali ${ }^{97}$. Non è un caso che uno dei motivi ricorrenti nella polemica tra le due città sia stato incentrato proprio sull'adeguatezza del Palazzo reale a consentire al viceré di svolgervi nel suo complesso le attività istituzionali. Anche per questa ragione a Messina si procedette per volontà del viceré Emanuele Filiberto di Savoia alla costruzione della monumentale Palazzata (1622), con l'intento preciso di rispondere all'esigenza di quella città di accreditarsi anche sul piano architettonico e delle infrastrutture come capitale del regno $^{98}$. Si trattava di una «fabbrica meravigliosa» che doveva riunire residenze e uffici pubblici e privati, «un'orchestra con simmetria e ordine di palazzi, che pare all'occhio d'essere un solo Palazzo»» ${ }^{99}$.

Ma c'era anche un problema di costi, dal momento che tutto l'apparato di corte era stimato pari a 70 o 80 mila scudi l'anno distribuiti in diverse voci ${ }^{100}$. A Palermo si nutrivano forti dubbi circa la possibilità di Messina di farsene carico, mentre d'altra parte il Senato cittadino aveva molto investito negli anni, sostenendo a proprie spese e indebitandosi la costruzione di edifici e infrastrutture conformi al suo ruolo di capitale, delle quali invece Messina era carente ${ }^{101}$. E si continuò sempre a insistere

\footnotetext{
${ }_{96}$ Maurizio Vesco, "Una strada, due regge, una mappa: la committenza di don García Álvarez de Toledo", Mediterranea-ricerche storiche 41 (2017): 566-584.

${ }^{97}$ Rossella Cancila, "Palermo e Messina: residenza viceregia e questione dei Tribunali nel dibattito secentesco", in Capitali senza re, 123-150.

${ }^{98}$ Per una sintesi su questo argomento, cfr. il recente saggio di Sabina Montana, "Emanuele Filiberto di Savoia committente di architettura in Sicilia (1622-1624)", in La Sicilia dei viceré nell'età degli Asburgo, 187-204. Per gli interventi precedenti sul Palazzo reale di Messina al tempo del viceré Toledo, cfr. Vesco, "Una strada": 558-566.

${ }^{99}$ Così Placido Samperi nel 1644, cit. in Montana, "Emanuele Filiberto di Savoia", 190.

100 Memoriale de la Deputazione del Regno di Sicilia e de la Città di Palermo intorno a la divisione di quel Regno, che tenta la città di Messina (Palermo: per Alfonzo dell'Isola, 1630), cc. non numerate.

101 Anche Modesto Gambacorta nella sua relazione del 1593 aveva segnalato una maggiore capacità ricettiva di Palermo: gli abitanti delle altre città «con più facilità, commodità e manco spesa vengono a Palermo, et ivi possono condurre le vittovaglie e sue mercantie e robbe; et ivi si tratterranno con maggior
} 
sull'inopportunità della residenza a Messina: il trasferimento della sede dei Tribunali avrebbe comportato infatti anche lo spostamento di «un numero innumerabile di dottori, procuratori, agenti, sollecitatori, portieri et altri ministri inferiori, necessari così al tratto, come all'esecution di negotii». $\mathrm{E}$ ne sarebbe risultato un rallentamento dell'esercizio giurisdizionale, con l'inevitabile sospensione dell'attività pubblica e privata e il rallentamento dell'attività di governo ${ }^{102}$. Insomma, «se in que' crepuscoli di residenza mancherà la corte a negotii, mancheranno altresì i negotii alla corte $[\ldots] \mathrm{Et}$ in quell'otio di foro mancheranno a gli avvocati clienti et a' clienti gli avvocati» ${ }^{103}$.

Col trasferimento materiale della corte, tribunali, fori, uffici, curiali, si sarebbero messi in movimento migliaia di uomini con famiglie, donne e bambini al seguito, «et altri ancora, che mercadantano con la Corte», costretti ad affrontare i pericoli di un viaggio difficoltoso anche per la minaccia rappresentata dagli attacchi dei corsari di Tunisi e Barberia. A ciò si aggiunga la difficoltà a trovare casa per l'incapacità di Messina a ospitare il nuovo flusso di gente, in quanto la scarsità di pietra rendeva caro e difficile costruirvi («cara la fabbrica e poco grata a' forastieri la stanza»). Appena avrebbero potuto dimorarvi i ministri, ma che sarebbe stato poi degli ufficiali inferiori? dei dottori e di tutta la «bassa curialità»?

Si insisteva inoltre sul problema determinato dallo smembramento degli incartamenti («si è sempre provato sì gran danno che lo scommuoverli de’ loro archivii e luoghi ordinati, è stato più volte prohibito da' capitoli, prammatiche e altre ordinattioni reali») e dai rischi determinati dal loro trasporto per via mare e per via terra ${ }^{104}$. I dottori non avrebbero a disposizione le loro "librarie" per difendere in maniera opportuna i propri clienti. E inoltre, dove sistemare $i$ tribunali che hanno bisogno di molte stanze? Le sontuose fabbriche già predisposte a Palermo sarebbero rimaste vuote, aspettando che a Messina si fabbricasse «nuovo albergo alla corte». Le diverse attività si sarebbero disperse per la città a causa della mancanza di spazi con disagio per i negozianti e per gli ufficiali, mentre invece a Palermo tutto si era concentrato nei secoli attorno al Palazzo reale.

Ne emerge un dato assai interessante, quello dell'impatto della corte sul tessuto urbano, in termini sia di servizi e infrastrutture a livello urbanistico sia anche di ricadute sul piano economico e sociale. La corte non era insomma un affare che riguardava soltanto le élites politiche, culturali, sociali, ma connetteva a sé un indotto economico e sociale di primaria importanza: essa non era solo un centro di spesa e di consumo, ma costituiva un potente stimolo alle attività produttive, quelle legate al circuito delle

commodo per la fertilità del paese e per trovarsi in questa città commodità d'alberghi, e molti spedienti per poter vivere e occuparsi in diversi essercizii e negozi». Al contrario invece Messina, posta in una punta e promontorio troppo distante dal «corpo del Regno» non offriva «commodità alcuna ai forastieri» (BCPa, ms. Qq F 76, cc. nn.: Discorso di Don Modesto Gambacorta marchese de la Motta, Presidente del Real Patrimonio di Sicilia, e regente che fu di poi di quel Regno in Ispagna, al Conte D'Olivares viceré e capitan generale di Sicilia sopra la residenza alternativa de la Corte in Palermo e Messina, Palermo 19 febbraio 1593).

102 Reina, L'Idra dicapitata, 17-19.

103 Ibidem, 19.

104 Ibidem, 36. Sul pericolo dello smembramento degli archivi, che «d'antichissimi e immemorabili tempi sono stati fondati e stabiliti a Palermo e non in altra parte», e sui rischi del loro trasporto in altre sedi aveva già messo in guardia anche Modesto Gambacorta (Discorso di Don Modesto Gambacorta). 
botteghe artigiane, ma anche alla cantieristica urbanistico-architettonica ${ }^{105}$. Alimentava domanda, committenza, generava bisogni e dunque creava lavoro e servizi: «un motore di ridistribuzione di risorse finanziarie e umane assolutamente senza eguali per l'economia urbana preindustriale» ${ }^{106}$.

${ }^{105}$ Cfr. le considerazioni di Fantoni, "La corte", 112.

106 Marcello Fantoni, Il potere dello spazio. Principi e città nell'talia dei secoli XV-XVII (Roma: Bulzoni, 2002), 216. 


\section{BIBLIOGRAFIA DI RIFERIMENTO}

Alvarez-Ossorio Alvariño, Antonio, "Corte y provincia en la Monarquía Católica: la corte de Madrid y el Estado de Milán, 1660-1700", in La Lombardia spagnola: nuovi indirizæi di ricerca, a cura di Elena Brambilla e Giovanni Muto (Milano: Unicopli, 1997), 283-341.

Antonelli, Attilio, a cura di, Cerimoniali della corte di Napoli, 5 Voll. (Napoli: Arte'm, 20122019).

Baviera Albanese, Adelaide, "I ventisette giorni di 'governo' nel Regno di Sicilia di Eleonora De Moura y Moncada marchesa di Castel Rodrigo (16 aprile-13 maggio 1677)", Archivio Storico Siciliano - serie IV 24 (1998): 267-301.

—, "L'ufficio del Consultore del viceré nel quadro delle riforme dell'amministrazione giudiziaria del sec. XVI in Sicilia", in Scritti minori, a cura di Adelaide Baviera Albanese (Soveria Mannelli: Rubbettino, 1993), 111-158.

—, Diritto pubblico e istiturioni amministrative in Sicilia. Le fonti (Roma: Il Centro di ricerca, 1981).

Baviera Albanese, Adelaide, a cura di, Los avertimientos del doctor Fortunato sobre el govierno de Sicilia (1591) (Palermo: Società Siciliana per la Storia Patria, 1976).

Bazzano, Nicoletta, Palermo fastosissima. Cerimonie cittadine in età spagnola (Palermo: Palermo University Press, 2016).

—, "Pietraperzia Branciforte Barresi, Francesco", Diæionario Biografico degli Italiani, vol. 83 (2015), on-line: https://www.treccani.it/enciclopedia/pietraperziabranciforte-barresi-francesco_(Dizionario-Biografico)/ (ultima consulta: 08/11/2021).

—, Marco Antonio Colonna (Roma: Salerno, 2003).

Barni, Gianluigi, "Dissensi fra i segretari del Gran Cancelliere e i segretari del Governatore a Milano", Archivio Storico Lombardo 40 (1933): 516-527.

Benigno, Francesco, "A patti con la monarchia degli Asburgo? La Sicilia spagnola tra integrazione e conflitto", in Studi storici dedicati a Orazio Cancila, a cura di Antonino Giuffrida, Fabrizio D’Avenia e Daniele Palermo (Palermo: Quaderni di Mediterranea-ricerche storiche, 2011), vol. I, 373-392.

—, L’ombra del re. Ministri e lotta politica nella Spagna del Seicento (Venezia: Marsilio, 1992). 
—, La Sicilia dei viceré. Potere e conflitto nella Sicilia spagnola (sec. XVI-XVIII) (Palermo: Palermo University Press, 2017).

—, Una casa, una terra. Ricerche su Paceco, paese nuovo nella Sicilia del Sei e Settecento (Catania: CUECM, 1985).

Bertelli, Sergio, "La corte come problema storiografico. A proposito di alcuni libri (più o meno) recenti”, Archivio Storico Italiano 164/1 (2006): 129-163.

Brambilla, Elena e Muto, Giovanni, a cura di, La Lombardia spagnola: nuovi indirizzi di ricerca (Milano: Unicopli, 1997).

Burgarella, Pietro, “Le carte della «Commissaria di mezz'annata» nell'Archivio di Stato di Palermo", Rassegna degli Archivi di Stato 33 (1973): 331-358.

—, "Verbali del Sacro Regio Consiglio di Sicilia del secolo XV", Archivio storico siciliano - serie IV 7 (1981): 115-210.

Cancila, Orazio, I Ventimiglia di Geraci (1258-1619) (Palermo: Quaderni di Mediterranea-ricerche storiche, 2016).

Cancila, Rossella, "Corsa e pirateria nella Sicilia della prima età moderna", Quaderni storici 2 (2001): 363-378.

—, a cura di, Capitali senza re nella Monarchia spagnola. Identità, relarioni, immagini (secc. XVI-XVIII), (Palermo: Quaderni di Mediterranea-ricerche storiche, 2020).

—, "Palermo e Messina: residenza viceregia e questione dei Tribunali nel dibattito secentesco", in Capitali senza re nella Monarchia spagnola. Identità, relazioni, immagini (secc. XVI-XVIII), a cura di Rosella Cancila (Palermo: Quaderni di Mediterranea-ricerche storiche, 2020), 123-150.

—, Fisco ricchezza comunità nella Sicilia del Cinquecento (Roma: Istituto Storico Italiano, 2001).

Cantù, Francesca, a cura di, Las Cortes Virreinales de la Monarquía Española (Roma: Viella, 2008).

Capasso, Gaetano, Il governo di don Ferrante Gonzaga in Sicilia dal 1535 al 1543 (Palermo: Scuola Tip. «Boccone del povero», 1905).

Cisneros, Pedro de, Relación de las cosas del Reyno de Sicilia, edizione a cura di Vittorio Sciuti Russi (Napoli: Jovene, 1990). 
Corsetto, Pietro, "Instruccion para el principe Filiberto quando fue al Virreynato de Sicilia", in Il governo della Sicilia in due Relazioni del primo Seicento, a cura di Vittorio Sciuti Russi (Napoli: Jovene, 1984).

Cremonini, Cinzia, Il Consiglio Segreto tra "interim" e prassi quotidiana (1622-1706), in La Lombardia spagnola: nuovi indirizzi di ricerca, a cura di Elena Brambilla e Giovanni Muto (Milano: Unicopli, 1997), 225-261.

-, Alla corte del governatore. Feste, riti e cerimonie a Milano tra XVI e XVIII secolo (Roma: Bulzoni, 2012).

Crivella, Alfonso, Trattato di Sicilia (1593), edizione a cura di Adelaide Baviera Albanese (Caltanissetta-Roma: Sciascia editore, 1970).

De Nardi, Loris, Oltre il cerimoniale dei viceré. Le dinamiche istituzionali nella Sicilia barocca (Padova: libreriauniversitaria.it edizioni, 2014).

Di Blasi, Giovanni Evangelista, Storia cronologica de' Viceré, edizione a cura di Illuminato Peri (Palermo: Edizioni della Regione Siciliana, 1974).

Di Castro, Scipio, Avvertimenti a Marco Antonio Colonna quando andò viceré di Sicilia, edizione a cura di Armando Saitta, (Roma: Edizioni di Storia e Letteratura, 1959).

Di Giovanni, Vincenzo, Del Palermo restaurato, edizione a cura di Mario Giorgianni e Antonio Santamaura (Palermo: Sellerio, 1989).

Dollo, Corrado, Modelli scientifici e filosofici nella Sicilia spagnola (Napoli: Guida, 1984).

Fantoni, Marcello, "La corte", in Le parole che noi usiamo: categorie storiografiche e interpretative dell'Europa moderna, a cura di Amedeo Quondam e Marcello Fantoni (Roma: Bulzoni, 2008), 109-141.

—, a cura di, The Court in Europe (Roma: Bulzoni, 2012).

—, Il potere dello spazio. Principi e città nell'Italia dei secoli XV-XVII (Roma: Bulzoni, 2002).

Favarò, Valentina, Carriere in movimento. Francisco Ruiz de Castro e la monarchia di Filippo III (Palermo: Studi e ricerche - Mediterranea - ricerche storiche, 2013).

Giardina, Camillo, L'istituto del Viceré di Sicilia (1415-1798) (Palermo: Boccone Del Povero, 1930). 
Giuffrida, Antonino, "La fortezza indifesa e il progetto del Vega per una ristrutturazione del sistema difensivo siciliano", in Mediterraneo in armi (secc. XV-XVIII), a cura di Rossella Cancila (Palermo: Quaderni di Mediterranea-ricerche storiche, 2007), 227-288.

—, La finanza pubblica_nella Sicilia del '500 (Caltanissetta-Roma: Sciascia editore, 1999).

Gonzáles Reyes, Carlos, "Il governo di don Bernardino de Cárdenas, III duca di Maqueda, nella Sicilia di fine Cinquecento: potere e architettura", in La Sicilia dei viceré nell'età degli Asburgo (1516-1700), a cura di Stefano Piazza (Palermo: Edizioni Caracol, 2016), 169-185.

Gonzáles Tornel, Pablo, "Il duca d'Uzeda, la duchessa e l'apoteosi della festa barocca", in La Sicilia dei viceré nell'età degli Asburgo (1516-1700), a cura di Stefano Piazza (Palermo: Edizioni Caracol, 2016), 247-258.

Hernando Sánchez, Carlos José, "Los virreyes de la Monarquía española en Italia. Evolución y práctica de un oficio de gobierno", Studia historica. Historia moderna 26 (2004): 43-73.

—, Castilla y Nápoles en el siglo XVI. El virrey Pedro de Toledo (Valladolid: Junta de Castilla y León, 1994).

Koenigsberger, Helmut G., L'esercizio dell'impero (Palermo: Sellerio, 1997).

Ligresti, Domenico, Le armi dei Siciliani Cavalleria, guerra e moneta nella Sicilia spagnola (secoli XV-XVII) (Palermo: Ebook Mediterranea-ricerche storiche, 2013).

-, Sicilia aperta. Mobilità di nomini e idee nella Sicilia spagnola (secoli XV-XVII) (Palermo: Quaderni di Mediterranea-ricerche storiche, 2006).

—, "Le piccole corti aristocratiche nella Sicilia spagnola", Archivio Storico per la Sicilia orientale 94/1 (1998): 11-35.

Mafrici, Mirella, a cura di, Alla corte napoletana. Donne e potere dall'età aragonese al viceregno austriaco 1442-1734 (Napoli: Fridericiana editrice universitaria, 2012).

Manfrè, Valeria e Velasco, Margarita Martín, "La corte virreinal siciliana del IV Duque de Uceda en Sicilia (1687-1696)" in En tierra de confluencias. Italia y la Monarquía de España, siglos XVI-XVIII, a cura di Cristina Bravo Lozano e Roberto Quirós Rosado (Valencia: Albatros Ediciones, 2013), 61-79.

Marrone, Antonino, Bivona città feudale (Caltanissetta-Roma: Sciascia editore, 1987). 
Martínez Millán, José, "La corte de la Monarquia Hispánica”, Studia historica. Historia moderna 28 (2006): 17-61.

Martínez Millán, José; Gonzáles Cuerva, Rubén e Rivero Rodríguez, Manuel, a cura di, La Corte de Felipe IV (1621-1665). Reconfiguración de la Monarquía Católica, tomo IV, vol. III (Madrid: Polifemo, 2018).

Masbel, Bernardino, Descrizione e relatione del governo di stato e guerra del regno di Sicilia (Palermo: Pietro Coppola, 1694).

Mateu Ibars, Josefina, "«Noticias del Reyno de Sicilia y govierno para los virreys». Manuscrito de la Biblioteca Comunale de Palermo", Boletín de la Real Academia de Buenas Letras de Barcelona 30 (1963-64): 183-240.

Mauro, Ida, Spazio urbano e rappresentazione del potere: le cerimonie della città di Napoli dopo la rivolta di Masaniello (1648-1672) (Napoli: FedOAPress, 2020).

Mazzarese Fardella, Enrico, Cerimoniale de' Signori Vicerè (1584-1688) (Palermo: Società Siciliana per la Storia Patria 1976

Mongitore, Antonino, Parlamenti generali ordinarij et straordinarj celebrati nel Regno di Sicilia dal 1494 fino al 1658 (Palermo: presso Pietro Bentivegna, 1717).

Montana, Sabina, "Emanuele Filiberto di Savoia committente di architettura in Sicilia (1622-1624)", in La Sicilia dei viceré nell'età degli Asburgo (1516-1700), a cura di Stefano Piazza (Palermo: Edizioni Caracol, 2016), 187-204.

Mozzarelli, Cesare e Venturi, Gianni, a cura di, L' Europa delle corti alla fine dell' antico regime (Roma: Bulzoni, 1991).

Musi, Aurelio, L’impero dei viceré (Bologna: il Mulino, 2013).

Muto, Giovanni, "Capital y Corte en la Nápoles española”, Reales Sitios 40 (2003): 315.

Novi Chavarria, Elisa, "Corte e viceré di Napoli nell'età di Filippo IV", in La Corte de Felipe IV (1621-1665). Reconfiguración de la Monarquía Católica, a cura di José Martínez Millán, Rubén González Cuerva e Manuel Rivero Rodríguez (Madrid: Polifemo, 2018), tomo IV, vol. III, 1307-1333.

O’Malley, John W., I primi Gesuiti, (Milano: Vita e pensiero, 1999).

Petronio, Ugo, Il Senato di Milano._Istituzioni giuridiche ed eserciz̨io del potere nel Ducato di Milano da Carlo V a Giuseppe II (Milano: Giuffrè, 1972). 
Piazza, Stefano, a cura di, La Sicilia dei viceré nell'età degli Asburgo (1516-1700) (Palermo: Edizioni Caracol, 2016).

—, "Volontà governative e poteri locali nel rinnovamento urbano di Palermo", in Capitali senza re nella Monarchia spagnola. Identità, relazioni, immagini (secc. XVIXVIII), a cura di Rossella Cancila (Palermo: Quaderni di Mediterranea-ricerche storiche, 2020), 151-163.

Pilo, Rafaella, Luigi Guglielmo Moncada e il governo della Sicilia (1635-1639) (CaltanissettaRoma: Sciascia editore, 2008).

Reina, Placido (Idoplare Copa), L'Idra dicapitata (Vicenza: per Gio. Antonio Gabucci, 1662).

Rivero Rodríguez, Manuel, La edad de oro de los virreyes: el virreinato en la monarquía bispánica durante los siglos XVI y XVII (Madrid: Ediciones Akal, 2011).

Salomone-Marino, Salvatore, "La congregazione dei Cavalieri d'armi e le pubbliche giostre in Palermo nel secolo XVI. Notizie e documenti", Nuove effemeridi siciliane - serie III 5 (1877): 103-139.

Scalisi, Lina e Foti, Rita Loredana, "Il governo dei Moncada (1567-1672)", in La Sicilia dei Moncada. Le corti, l'arte e la cultura nei secoli XVI-XVII, a cura di Lina Scalisi (Catania: Domenico Sanfilippo Editore, 2006), 15-58.

Scalisi, Lina, "Magnus Siculus". La Sicilia tra impero e monarchia (1513-1578) (Roma-Bari: Laterza, 2012).

—, Da Palermo a Colonia. Carlo Aragona Tagliavia e la questione delle Fiandre (1577-1580) (Roma: Viella, 2019).

Sciuti Russi, Vittorio, a cura di, Il Parlamento del 1612. Atti e documenti (Catania: Bonanno, 1984).

_, Astrea in Sicilia (Napoli: Jovene, 1983).

—, Mario Cutelli. Una utopia di governo (Catania: Bonanno, 1994).

Signorotto, Gianvittorio, a cura di, Ferrante Gonzaga. Il Mediterraneo, l'Impero (1507-1557) (Roma: Bulzoni, 2009).

—, "L'Italia degli Austrias._Monarchia cattolica e domini italiani nei secoli XVI e XVII", numero monografico della rivista Cheiron 17-18 (1992). 
—, Milano spagnola. Guerra, istituðৃioni, uomini di governo (1635-1660) (Firenze: Sansoni, 1996).

Sodano, Giulio, "Le aristocrazie napoletane ai tempi di Filippo IV", in La Corte de Felipe IV (1621-1665). Reconfiguración de la Monarquía Católica, a cura di José Martínez Millán, Rubén Gonzáles Cuerva e Manuel Rivero Rodríguez (Madrid: Polifemo, 2018), tomo IV, vol. III, 1335-1380.

Tricoli, Giuseppe, Un periodo del governo spagnolo di Sicilia nella Relazione del viceré Uzeda (1687-1696) (Palermo: Edizioni Thule, 1980).

Vannugli, Antonio, "Il segretario Juan de Lezcano e la sua collezione di dipinti italiani", in Centros de poder italianos en la monarquia hispánica (siglos XV-XVIII), a cura di José Martínez Millán e Manuel Rivero Rodríguez (Madrid: Polifemo, 2010), vol. III, 1487-1542.

Vergara, Francesco, Il Parlamento del 1615. Atti e documenti (Catania: Bonanno, 1991).

Vesco, Maurizio, "La Regia Razza di cavalli e le scuderie monumentali nella Sicilia degli Asburgo: il modello "negato" delle cavallerizze dei Palazzi Reali di Palermo e Messina", in Las caballerizas reales y el mundo d caballo, a cura di Juan Aranda Doncel e José Martínez Millán (Cordoba: Instituto Universitario "La Corte e Europa”, 2016), 391-428.

—, "Un regno, due capitali. Opere pubbliche, politica dell'immagine e costruzione simbolica a Palermo e Messina (secc. XVI-XVII)", in Capitali senza re nella Monarchia spagnola. Identità, relazioni, immagini (secc. XVI-XVIII), a cura di Rossella Cancila (Palermo: Quaderni di Mediterranea-ricerche storiche, 2020), 339-367.

—, "Una strada, due regge, una mappa: la committenza di don García Álvarez de Toledo", Mediterranea-ricerche storiche 41 (2017): 543-592.

Visceglia, Maria Antonietta, "Corti italiane e storiografia europea. Linee di lettura", in L'Italia alla fine del Medioevo: $i$ caratteri originali nel quadro europeo, a cura di Federica Cengarle (Firenze: Firenze University Press, 2006), vol. II, 37-85.

Zapperi, Roberto, “Arduino, Andrea”, Dizionario Biografico degli Italiani, vol. 4 (1962), on-line: https://www.treccani.it/enciclopedia/andrea-arduino_(DizionarioBiografico)/ (ultima consulta: 08/11/2021). 
—, "Avalos, Francesco Ferdinando, marchese di Pescara", Diæionario Biografico degli Italiani, vol. 4 (1962), on-line: https://www.treccani.it/enciclopedia/avalosfrancesco-ferdinando-marchese-di-pescara_(Dizionario-Biografico)/ (ultima consulta: 08/11/2021).

Recibido: 28 de mayo de 2021

Aceptado: 19 de julio de 2021 